\title{
A Survey on \\ OFDM-Based Elastic Core Optical Networking
}

\author{
Guoying Zhang ${ }^{12}$, Marc De Leenheer ${ }^{13}$, Annalisa Morea ${ }^{4}$, and Biswanath Mukherjee ${ }^{1}$ \\ ${ }^{1}$ University of California - Davis, USA \\ ${ }^{2}$ China Academy of Telecom Research, China \\ ${ }^{3}$ Ghent University - IBBT, Belgium \\ ${ }^{4}$ Alcatel-Lucent Bell Labs, France \\ Email: \{zguoying, mleenheer,bmukherjee\}@ucdavis.edu \\ zhangguoying@catr.cn \\ annalisa.morea@alcatel-lucent.com
}

November 2011 


\section{Contents}

Abstract.

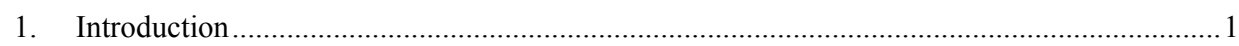

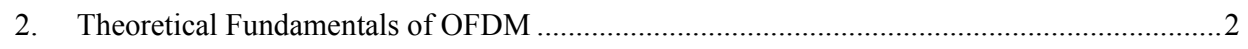

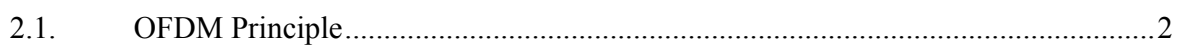

2.2. Building Blocks of OFDM Systems.................................................................... 4

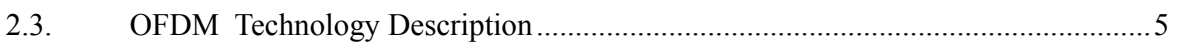

2.3.1. Guard Interval and Cyclic Prefix..................................................................

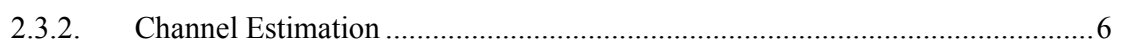

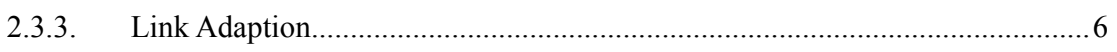

2.4. Advantages and Disadvantages of OFDM ........................................................

3. Optical OFDM Transmission Technology ............................................................................ 7

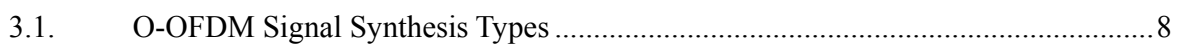

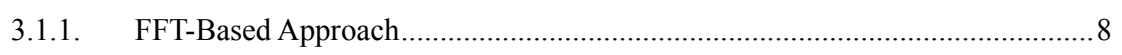

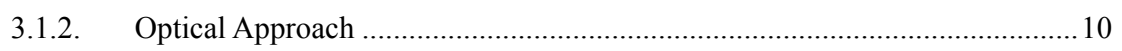

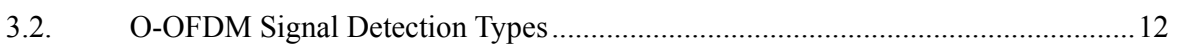

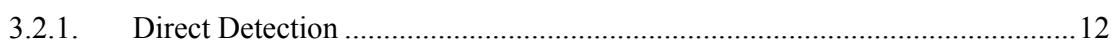

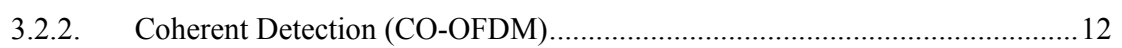

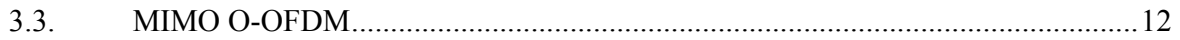

3.4. Modulation Formats and Adaptive Modulation ................................................. 15

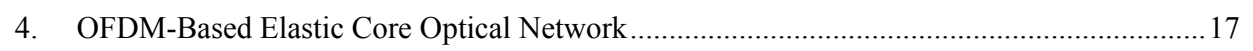

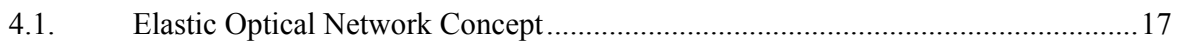

4.2. OFDM-Based Elastic Optical Network Architecture ............................................. 19

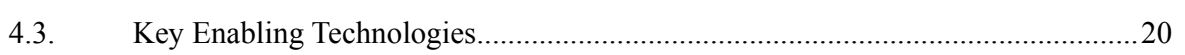

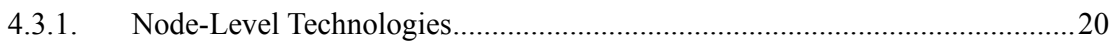

4.3.1.1. Data-Rate/Bandwidth-Variable Transponder..................................20

4.3.1.2. Bandwidth-Variable Optical Switching ............................................22

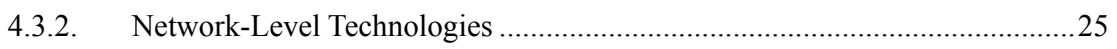

4.3.2.1. Flexible Spectrum Slot Specification ……......................................26

4.3.2.2. Routing and Spectrum Allocation Algorithm ..................................22

4.3.2.2.1. Static RSA with ILP (Integer Linear Programing) ..........................2 27

4.3.2.2.2. Heuristic Algorithms for Static and Dynamic RSA.........................2 28

4.3.2.2.3. RSA for Survivable Networks ........................................................29

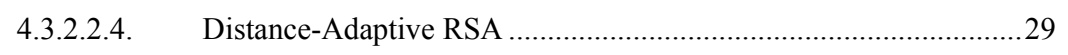

4.3.2.2.5. RSA for Time-Varying Traffic ...................................................... 31

4.3.2.2.6. Network Defragmentation RSA ……............................................... 31

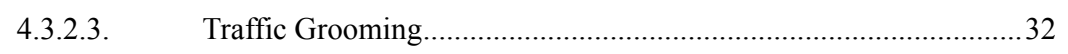

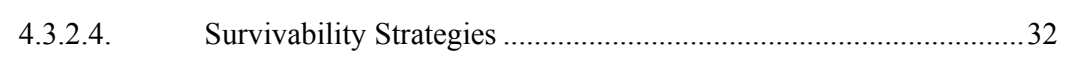

4.3.2.5. Optical Network Virtualization ........................................................ 33 


\begin{tabular}{|c|c|c|}
\hline & 4.3.2.6. & Energy Efficiency ...................... \\
\hline & 4.3.2.7. & 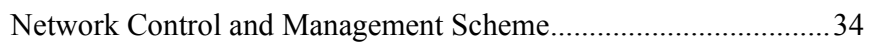 \\
\hline 5 . & Conclusion . & $\ldots 34$ \\
\hline 6 & eference & \\
\hline 7 & Acronym & .. 43 \\
\hline
\end{tabular}




\begin{abstract}
Orthogonal frequency-division multiplexing (OFDM) is a modulation technology that has been widely adopted in many new and emerging broadband wireless and wireline communication systems. Due to its capability to transmit a high-speed data stream using multiple spectral-overlapped lower-speed subcarriers, OFDM technology offers superior advantages of high spectrum efficiency, robustness against inter-carrier and inter-symbol interference, adaptability to server channel conditions, etc. In recent years, there have been intensive studies on optical OFDM (O-OFDM) transmission technologies, and it is considered a promising technology for future ultra-high-speed optical transmission. Based on O-OFDM technology, a novel elastic optical network architecture with immense flexibility and scalability in spectrum allocation and data rate accommodation could be built to support diverse services and the rapid growth of Internet traffic in the future. In this paper, we present a comprehensive survey on OFDM-based elastic optical network technologies, including basic principles of OFDM, O-OFDM technologies, the architectures of OFDM-based elastic core optical networks, and related key enabling technologies. The main advantages and issues of OFDM-based elastic core optical networks that are under research are also discussed.
\end{abstract}

Keywords: Optical Orthogonal Frequency-Division Multiplexing (O-OFDM), Elastic Optical Network, Data Rate/Bandwidth-Variable Transponder, Bandwidth-Variable Wavelength CrossConnect (BV-WXC), Routing and Spectrum Allocation (RSA), Traffic Grooming, Survivability, Network Virtualization.

\section{Introduction}

In recent years, Internet traffic in the core network has been doubling almost every two years, and predictions indicate that it will continue to exhibit exponential growth due to emerging applications such as high-definition and real-time video communications [1][2]. As a result of this rapid increase in traffic demands, large-capacity and cost-effective optical fiber transmission systems are required for realizing future optical networks. So far, Wavelength-Division Multiplexing (WDM) systems with up to $40 \mathrm{~Gb} / \mathrm{s}$ capacity per channel have been deployed in backbone networks, while 100 $\mathrm{Gb} / \mathrm{s}$ interfaces are now commercially available and $100 \mathrm{~Gb} / \mathrm{s}$ deployment are expected soon. Moreover, it is foreseen that optical networks will be required to support $\mathrm{Tb} / \mathrm{s}$ class transmission in the near future [2][3]. However, scaling to the growing traffic demands is challenging for conventional optical transmission technology as it suffers from the electrical bandwidth bottleneck limitation, and the physical impairments become more severe as the transmission speed increases [3].

On the other hand, emerging Internet applications such as Internet Protocol television (IPTV), video on demand, and cloud and grid computing applications demonstrate unpredictable changes in bandwidth and geographical traffic patterns [4]. This calls for a more data- rate flexible, agile, reconfigurable, and resource-efficient optical network, while the fixed and coarse granularity of current WDM technology will restrict the optical network to stranded bandwidth provisioning, inefficient capacity utilization, and high cost.

To meet the needs of the future Internet, the optical transmission and networking technologies are moving forward to a more efficient, flexible, and scalable direction. Solutions such as optical packet switching (OPS) and optical burst switching (OBS) that meet these requirements have been studied in the past few years, but cannot be considered as a near-term solution due to their immaturity [5].
批注 [gyzhang2]: [Authors' Comments] We have modified this sentence to emphasis the scope of this paper.

批注 [gyzhang3]: Reviewer1/Com ment 6a) Page $1: 40 \mathrm{~Gb} /=>$ up to 40 $\mathrm{Gb} / \mathrm{s}$.

[Authors' Response:] We have updated it. 
Recently, OFDM (Orthogonal Frequency-Division Multiplexing) has been considered a promising candidate for future high-speed optical transmission technology. OFDM is a multi-carrier transmission technology that transmits a high-speed data stream by splitting it into multiple parallel low-speed data channels. OFDM first emerged as a leading physical-layer technology in wireless communications, as it provides an effective solution to inter-symbol interference (ISI) caused by the delay spread of wireless channels. It is now widely adopted in broadband wireless and wireline networking standards, such as 802.11a/g Wi-Fi, 802.16 WiMAX, LTE (Long-Term Evolution), DAB and DVB (Digital Audio and Video Broadcasting), and DSL (Digital Subscriber Loop) around the world [3].

Because of the great success of OFDM in wireless and wireline systems, it is currently being considered for optical transmission and networking. With the intrinsic flexibility and scalability characteristics of optical OFDM technology (which will be described in Section 4.1 in more detail), a novel elastic optical network architecture, possessing the capability to manage signals with different data rate and variable bandwidth, can be built to meet the requirements of future optical networks [6].

In this paper, we present a comprehensive survey of OFDM-based optical high-speed transmission and networking technologies, with a specific focus on core optical network scenarios. We start with basic OFDM principles in Section 2, and introduce various kinds of optical OFDM transmission schemes and technologies in Section 3. Next, we address the OFDM-based elastic optical network, detailing its architecture and enabling technologies in Section 4. Finally, we present our concluding remarks in Section 5.

\section{Theoretical Fundamentals of OFDM}

\subsection{OFDM Principle}

OFDM is a special class of the Multi-Carrier Modulation (MCM) scheme that transmits a highspeed data stream by dividing it into a number of orthogonal channels, referred to as subcarriers, each carrying a relatively-low data rate [3]. Compared to WDM systems, where a fixed channel spacing between the wavelengths is usually needed to eliminate crosstalk, OFDM allows the spectrum of individual subcarriers to overlap because of its orthogonality, as depicted in Figure 1. Furthermore, the inter-symbol interference (ISI) of the OFDM signal can be mitigated as the per-subcarrier symbol duration is significantly longer than that of a single-carrier system of the same total data rate.
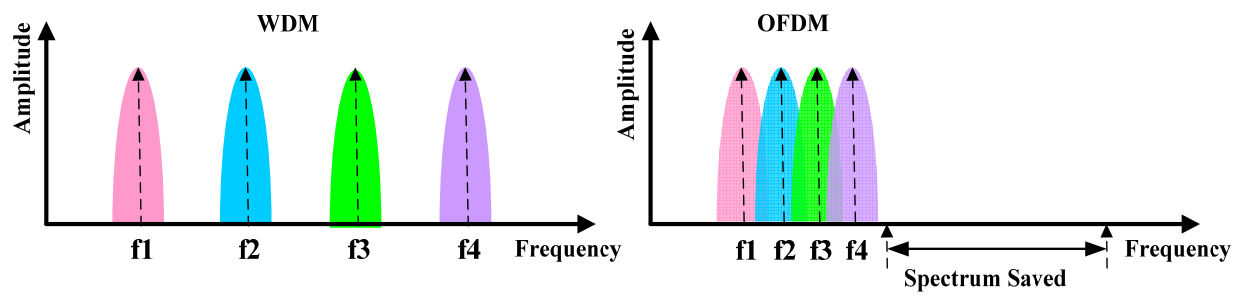

Figure 1 Spectrum of WDM signals and OFDM signal [7].

From the spectrum perspective, the orthogonal condition between multiple subcarriers is satisfied when their central frequencies are spaced $n / T_{s}$ apart, where $n$ is an integer and $T_{s}$ is the symbol duration. It can be seen in Figure 2(a) that the peak point of a subcarrier's spectrum corresponds to the 
zero point of other subcarriers' spectra. Therefore, when a subcarrier is sampled at its peak, all other subcarriers have zero-crossings at that point and thus do not interfere with the subcarrier being sampled. This orthogonality leads to a more efficient usage of spectral resources, which is limited for most communication media.

In the time domain, the OFDM signal is a synthesis of multiple subcarriers' waveforms, and consists of a continuous stream of OFDM symbols that have a regular symbol period, as shown in Figure 2(b).

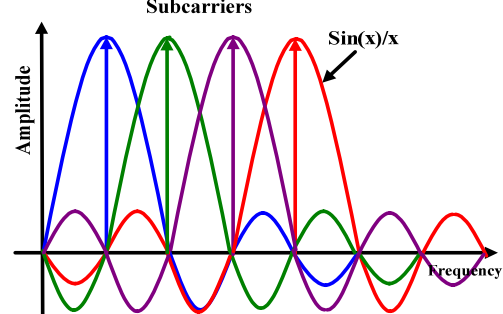

a) Frequency Domain

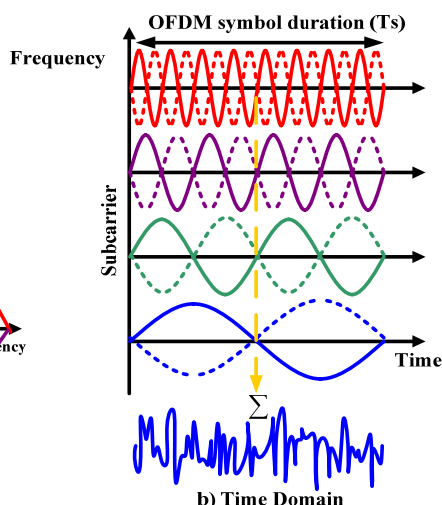

b) Time Domain

Figure 2 Spectrum and time domain expression of OFDM signal (with 4 subcarriers):

a) Spectrum domain; b) Time domain.

As mentioned above, OFDM is a special form of multi-carrier modulation with orthogonality between each subcarrier. A general multi-carrier modulation signal $s(t)$ is represented as [3]:

$$
\begin{aligned}
& s(t)=\sum_{i=-\infty}^{+\infty} \sum_{k=1}^{N_{s c}} c_{k i} s_{k}\left(t-i T_{s}\right) \\
& s_{k}(t)=\Pi(t) e^{j 2 \pi f_{k} t} \\
& \Pi(t)=\left\{\begin{array}{l}
1,\left(0<t \leq T_{s}\right) \\
0,\left(t \leq 0, t>T_{s}\right)
\end{array}\right.
\end{aligned}
$$

where $c_{k i}$ is the ith information symbol at thekth subcarrier, $s_{\mathrm{k}}$ is the waveform for the $k$ th subcarrier, $N_{s c}$ is the number of subcarriers, $f_{k}$ is the frequency of the subcarrier, $T_{s}$ is the symbol period, and $\Pi(t)$ is the pulse-shaping function. The detector for each subcarrier uses a filter that matches the subcarrier waveform. Therefore, the detected information symbol $c^{\prime}{ }_{k i}$ is given by:

$$
C^{\prime}{ }_{k i}=\int_{0}^{T_{s}} r\left(t-i T_{s}\right) s^{*}{ }_{k} d t=\frac{1}{T_{s}} \int_{0}^{T_{s}} r\left(t-i T_{s}\right) e^{-j 2 \pi f_{k}} d t
$$

where $r(t)$ is the received time-domain signal.

The orthogonal condition of an OFDM signal originates from a correlation between any two subcarriers, given by: 


$$
\delta_{k l}=\frac{1}{T_{s}} \int_{0}^{T_{s}} s_{k} s^{*}{ }_{l} d t=\frac{1}{T_{s}} \int_{0}^{T_{s}} e^{j 2 \pi\left(f_{k}-f_{l}\right) t} d t=e^{j \pi\left(f_{k}-f_{l}\right) T_{s}} \frac{\sin \left(\pi\left(\left(f_{k}-f_{l}\right) T_{s}\right)\right.}{\left(\pi\left(\left(f_{k}-f_{l}\right) T_{s}\right)\right.}
$$

If the condition:

$$
f_{k}-f_{l}=m \frac{1}{T_{s}}
$$

is satisfied, then the two subcarriers are orthogonal to each other. It can be seen that these orthogonal subcarrier sets, with their frequencies spaced at multiples of the inverse of the symbol periods, can be recovered with the matched filters in Eqn. (4) without inter-carrier interference, despite strong spectral overlapping.

It has been shown that OFDM modulation and demodulation can be implemented using inverse discrete Fourier transform (IDFT) and discrete Fourier transform (DFT), respectively [8]. The discrete value of the transmitted OFDM signal $s(t)$ is a N-point IDFT of the information symbol $c_{k}$, and the received information symbol $c^{\prime}{ }_{k}$ is a N-point DFT of the received sampled signal $r(t)$. To reduce the computational complexity of DFT/IDFT, efficient fast Fourier transform and inverse fast Fourier transform (FFT/IFFT) functions are normally used in OFDM systems to implement OFDM modulation and demodulation.

\subsection{Building Blocks of OFDM Systems}

A generic building block diagram of an OFDM system is shown in Figure 3. At the transmitter end, the input serial data stream is first converted into many parallel data streams through a serial-toparallel (S/P) converter, each mapped onto corresponding information symbols for the subcarriers within one OFDM symbol. Then, training symbols (TSs) are inserted periodically for channel estimation (which will be described in Section 2.3.2). These parallel data streams are modulated onto orthogonal subcarriers and converted to the time-domain OFDM signal, which is a two-dimensional complex signal including real and imaginary components, by applying the IFFT. Subsequently, a cyclic prefix is added into each OFDM symbol to avoid channel dispersion. The OFDM signal is then converted to analog by digital-to-analog conversion (DAC), and filtered with a low-pass filter (LPF) to remove the alias signal, yielding the OFDM baseband signal. The baseband signal can be up-converted to an appropriate radio frequency (RF) passband with an in-phase/quadrature-phase (IQ) modulator and a band-pass filter (BPF).

At the receiver end, the OFDM signal is down-converted to baseband with an IQ demodulator, sampled with an analog-to-digital converter (ADC), and then the complex-form OFDM signal is demodulated by a fast Fourier transform (FFT) function. The demodulated signals go through a symbol decision module, where synchronization, channel estimation, and compensation are performed before a symbol decision is made. Finally, multiple data channels are converted back to a single data stream by parallel-to-serial $(\mathrm{P} / \mathrm{S})$ operation. 


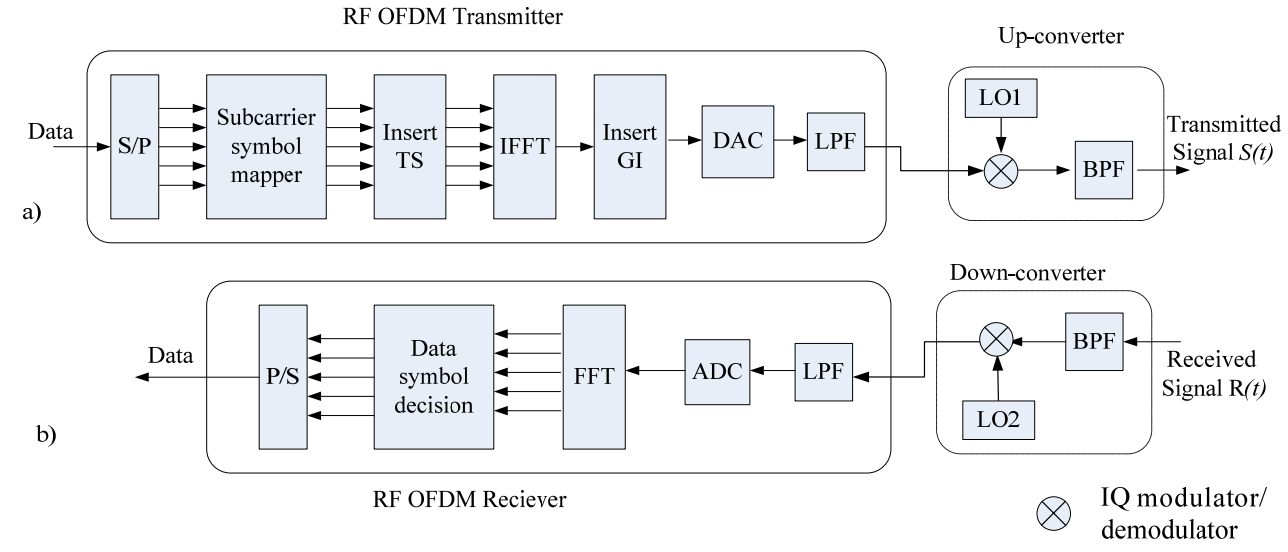

Figure 3 Building blocks of OFDM system: a) OFDM Transmitter; b) OFDM Receiver [3]. (S/P: Serial/Parallel; P/S: Parallel/Serial; TS: Training Symbols; FFT: Fast Fourier Transform; IFFT: Inverse Fast Fourier Transform; GI: Guard Interval; DAC: Digital-to-Analog Convertor; ADC: Analogto-Digital Convertor; LO: Local Oscillator; LPF: Low-Pass Filter; BPF: Band-Pass Filter; IQ: In-phase and Quadrature phase; RF: Radio Frequency)

\subsection{OFDM Technology Description}

\subsubsection{Guard Interval and Cyclic Prefix}

One of the enabling techniques for OFDM is the insertion of a guard interval (GI) and cyclic prefix (CP) [9]. In optics, the phase velocity of an optical pulse depends on its frequency. Different frequency components of an optical pulse travel with different speeds, so the optical pulse is spread out after transmission (i.e., delay spread). Because of this dispersion phenomenon, an OFDM symbol with a large delay spread after a long-distance transmission may cross its symbol boundary, leading to interference with its neighboring OFDM symbol, which is referred to as inter-symbol interference (ISI). Furthermore, because OFDM symbols of different subcarriers are not aligned due to the delay spread, the critical orthogonality condition for the subcarriers will be lost, resulting in an inter-carrier interference (ICI) penalty [3].

To deal with the ISI caused by channel delay spread, a guard interval is inserted into the OFDM symbol, as shown in Figure 4. It can be shown that, if the maximum delay spread of the transmission channel is smaller than the guard interval, the ISI can be perfectly eliminated. ICI can be reduced by introducing a cyclic prefix into the guard interval. The cyclic prefix is a copy of the past beginning of the current symbol at the end, and ensures that the complete OFDM symbol with the longer delay can also be received with the appropriate DFT window shifting [3]. 


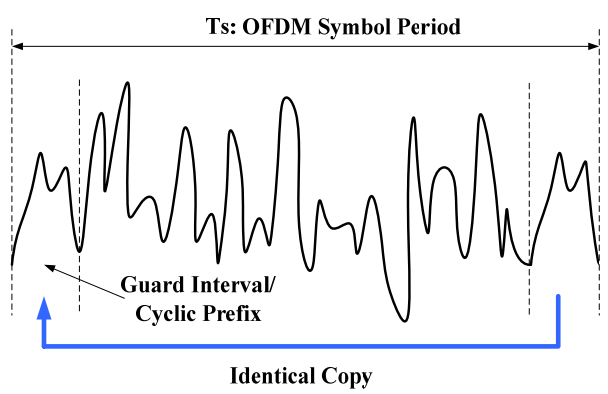

Figure 4 Guard Interval (GI) of an OFDM Symbol [3].

The length of the GI/CP is determined by the maximum delay spread induced by channel dispersion. Since the GI/CP introduces additional overhead, a conventional approach to minimizing this overhead is to set a long OFDM symbol interval, namely to use many subcarriers [10].

\subsubsection{Channel Estimation}

Similar to single-carrier modulation, time- and frequency-varying channels affect the performance of OFDM systems. The effect of the channel on the transmitted signal needs be estimated to recover the transmitted information. Many techniques have been proposed for estimating and adjusting both timing and frequency variation in OFDM systems [11]. The channel-state information can be estimated using non-blind channel estimation or blind channel estimation.

In non-blind channel estimation, training symbols (TS) containing information known by both the transmitter and the receiver are periodically inserted into data-bearing subcarriers. Channel-state information can be estimated based on these training symbols using channel-estimation algorithms. Sometimes, specific OFDM symbols with known data are inserted into selective subcarriers, called pilot subcarriers. The channel-state information corresponding to the pilot subcarriers is first estimated, and then the channel state corresponding to the data-bearing subcarriers can be obtained by interpolation. The overhead for training symbols or pilot subcarriers depends on the channel dynamics, where, in general, the more stable a channel is, the less overhead is required.

Blind channel estimation uses the intrinsic characteristics of the modulated signal, which is independent of the transmitted data, to estimate the channel state. This scheme requires careful design of the channel-estimation algorithm such that the system converges under all conditions. Compared to blind channel estimation, non-blind channel estimation is more straightforward, but introduces additional overhead.

\subsubsection{Link Adaption}

Link adaption is a widely-used technique to increase the spectral efficiency of broadband wireless data networks and digital subscriber lines. Link adaption exploits the frequency-selective nature of wideband channels. The basic idea is to adjust transmission parameters for each subcarrier, such as modulation and coding levels, according to certain channel conditions, to maximize the transmission data rate or minimize the transmission power.

For example, under good channel conditions, high-level modulation (i.e., more bit loading per symbol [12]) and less redundant error correction are used to increase throughput. In contrast, lowlevel modulation (i.e., less bit loading per symbol) and more redundant error correction are used under 
poor channel conditions, to ensure good transmission performance. These modulation formats will be described in more detail in Section 3.4

Link adaption is performed on a subcarrier basis, and is normally assisted by control signaling. Channel estimation is adopted to acquire the link condition, and subcarriers can be put on or off based on the link condition to guarantee communication.

\subsection{Advantages and Disadvantages of OFDM}

OFDM technology has a number of advantages that are key to future transmission systems, as indicated below.

(1) OFDM transmits a high-speed data stream by dividing it into multiple low-data- rate subcarriers, thereby increasing the symbol duration and reducing the inter-symbol interference. The intrinsic resilience to ISI makes OFDM a good candidate for future high-speed communication systems

(2) OFDM enables smooth upgrading from low-speed to high-speed transmission by simply augmenting the subcarriers and spectrum, without major changes in system design. Therefore, it is highly scalable for migration to the ever-increasing data rate in the future.

(3) High spectrum efficiency can be achieved by OFDM with overlapped subcarrier arrangement, so the system capacity can be greatly increased.

(4) The link-adaption capability of OFDM provides even higher spectrum efficiency, as distance and channel condition-adaptive modulation (bit per symbol adjustment) is employed.

(5) Energy-efficient operation to reduce power consumption can be implemented by an OFDM system through adaptive modulation and dynamically switching on/off specific subcarriers according to the channel condition and customer bandwidth requirement (which will be described in Section 4.3.2.6).

Besides its many advantages, OFDM has some disadvantages. One of its major challenges is the high peak-to-average power ratio (PAPR) caused by the symbol synthesis of multiple parallel subcarriers. This means that the transmitter and receiver components must have a wide dynamic range, such that the high PAPR signal will not be distorted. Another problem is that OFDM requires strict orthogonality between subcarriers, and thus is more sensitive to the frequency and phase noise that may interfere withd its orthogonality. These problems bring difficulties in system design, and are consequently a topic of intensive research.

\section{Optical OFDM Transmission Technology}

Because of the great success of OFDM in wireless and broadband access networks, it is being adopted as an optical transmission technique in recent years. Optical OFDM (O-OFDM) technology can be used in a range of optical communication systems including single-mode fiber (SMF) [13][14], multimode fiber (MMF) [15][16], plastic optical fiber (POF) [17], OFDM-PON [18], and optical wireless communication systems (OWC) [19][20]. In this paper, we mainly consider the single-mode fiber OFDM systems, to address the core optical network architecture discussed later in Section 4.

Currently, there are many different implementations of O-OFDM [21]. Various classifications exist to describe different O-OFDM schemes. In [3][22][23], two main forms of optical OFDM have been described as direct-detection optical OFDM (DD-OOFDM) and coherent optical OFDM (CO-OFDM),
批注 [gyzhang8]: Reviewer1/Comm ent 6 f) Page 6: Why is the OFDMA chapter needed? If it is to be included, it should be more elaborated. Otherwise it might be skipped.[Authors' Response:] The OFDMA part is not so relevent to the survey. We have deleted this section.

批注 [gyzhang9]: Reviewer2/Comm ent5.3) As a major candidate of the next generation optical network stru cture, it will be interesting to see the compatibility of PON network work ing with O-OFDM, etc. [3] Costeffective 33-Gbps intensity modulation direct detection multiband OFDM LR-PON system employing a 10-GHz-based transceiver

[Authors' Response:] We are aware of the trend of adopting O-OFDM technology in PON. We have added a reference to the suggested paper in the introduction part of Section 3 Because the scope of this survey is focusing on core optical network (as mentioned in the last paragraph in Section 1, and as reflected in the

批注 [gyzhang10]: Reviewer2/Com ment5.4) Although this paper is focusing on introducing the optical networking with single-mode fiber as data transmission media, the increasing interests in optical wireless communication (OWC) cannot be neglected. The O-OFDM on OWC has different features and requirements than normal fibers, such as the clipping distortion problem. It will be more comprehensive for this paper to briefly cover some related contont 
based on the signal detection technology used. In [10], three types of optical CO-OFDM system are classified to be FFT-Based CO-OFDM, All-Optical OFDM, and Electro-Optical OFDM, from both the signal synthesis and detection method perspectives.

In this paper, we describe optical OFDM schemes using two dimensions: signal synthesis mechanism (electrical and optical) and signal detection mechanism (direct detection and coherent detection), respectively, as depicted in Figure 5. Electro-Optical OFDM proposed in [10] was classified into the optical signal synthesis category.

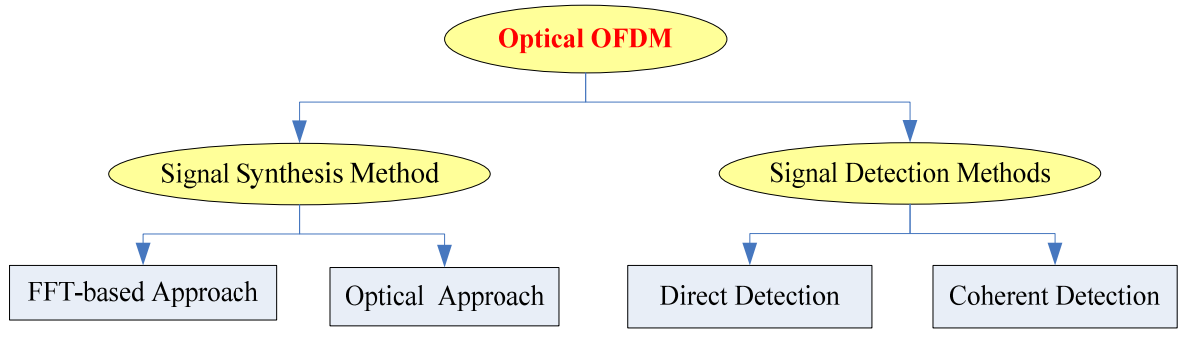

Figure 5 Types of O-OFDM.

\subsection{O-OFDM Signal Synthesis Types}

From the signal synthesis perspective, optical OFDM can be divided into two broad categories, namely the FFT-based approach (subcarriers generated in digital domain), and the optical approach (subcarriers generated in optical domain).

\subsubsection{FFT-Based Approach}

In the FFT-based approach, the OFDM subcarriers are generated in the digital domain using IFFT (Inverse Fast Fourier Transform). The FFT-based O-OFDM transmitter is composed of a radio frequency (RF) OFDM transmitter and a RF-to-optical up-converter, while the receiver is composed of an optical-to-RF down-converter, and a RF OFDM receiver [14].

The processing of the electrical OFDM carrier by an RF OFDM transmitter and receiver was described in Section 2.2. The function of the optical up-converter and down-converter is to modulate an OFDM baseband signal onto an optical carrier and vice versa. The conversion could be implemented with an intermediate frequency (IF) up-/down-conversion architecture or a direct up-/down-conversion architecture [3], as shown in Figure 6.

In the intermediate-frequency up-conversion architecture, as shown in Figure 6 a), the complexvalued OFDM signal is first up-converted to an intermediate frequency through in-phase (I) and quadrature $(\mathrm{Q})$ modulator, and then modulated onto the optical carrier through a conventional singleended Mach-Zehnder modulator (MZM). Besides the original baseband signal, an image-band is also generated with this method and stands side by side with the original baseband, and an optical band-pass filter (BPF) is needed to eliminate the image-band. For intermediate frequency down-conversion, the optical signal is first down-converted to an intermediate frequency, and then electrical I/Q detection is performed.

In the direct up-conversion architecture, as shown in Figure $6 \mathrm{~b}$ ), the optical transmitter uses a

批注 [gyzhang11]: Reviewer2/com
ment 4. 1) Page 10, line 30 and
Figure 6, ' three types of optical
CO-OFDM system are classified to
be FFT-Based CO-OFDM, All-
Optical OFDM, and Electro-Optical
OFDM', but in Figure 6, there are
only 'FFT-based approach' and
'Optical Approach'. Is ' Electro-
Optical OFDM ' sorted as in
'Optical Approach'? If so please
mention it.
[Authors' Response:] Electro-
Optical OFDM was indeed
classified as an 'Optical Approach'.
We have added the following
sentence to explain this."

批注 [gyzhang11]: Reviewer2/com CO-OFDM system are classified to be FFT-Based CO-OFDM, AllOFDM', but in Figure 6, there are only 'FFT-based approach' and 'Optical Approach'. Is ' ElectroOptical OFDM ' sorted as in mention it.

ence to explain this." 
complex Mach-Zehnder modulator, composed of two MZMs with 90-degree phase shift, to up-convert the real/imaginary parts of the complex OFDM signal from the electrical domain to the optical domain. For direct down-conversion, the OFDM optical receiver uses two pairs of balanced receivers and an optical 90-degree hybrid to perform optical I/Q detection. Such an optical conversion performs direct modulation of the OFDM signal onto the optical signal without image-band and thus no optical filtering is required at the transmitter.

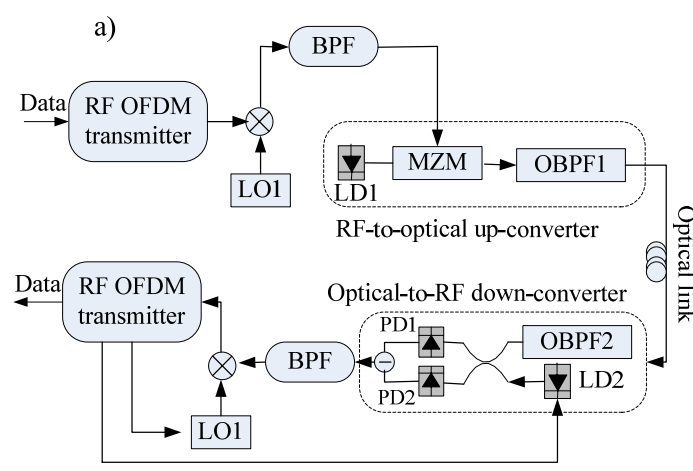

$\bigotimes$ RF I/Q modulator/demodulator

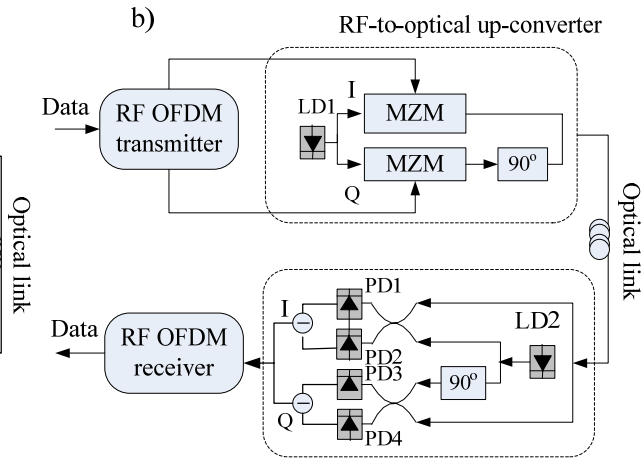

Optical-to-RF down-converter

Figure 6 Optical OFDM up/down conversion architecture: a) intermediate-frequency up-/downconversion; b) direct up-/down-conversion [22].

The electrically-generated subcarriers of OFDM can be modulated on a single optical carrier or on multiple optical carriers. These two schemes are described as single-band O-OFDM and multi-band OOFDM, respectively [24].

\section{(1) Single-Band OFDM}

Single-band OFDM modulates the electrical OFDM subcarriers on a single optical carrier, through the up-/down- conversion scheme discussed above. One of the main challenges of this single-band OFDM is that the operation speed of electronic devices such as the DAC/ADC and modulator drivers limits the transmission rate [25].

\section{(2) Multi-Band OFDM}

One approach to overcome the electrical processing bottleneck is to use the multi-band OFDM scheme [24][26][27][28], which generates a large number of electrical subcarriers and modulates them on multiple optical carriers, so that the data rate of each subcarrier can be reduced.

The basic principle of multi-band OFDM is to split the OFDM signal into multiple subbands, each modulated to an optical carrier, while maintaining their orthogonal property. As shown in Figure 7 , the entire OFDM spectrum comprises $N$ OFDM bands, each with the subcarrier spacing of $\Delta f$ and band frequency guard spacing of $\Delta f_{G}$. When the guard band spacing $\left(\Delta f_{G}\right)$ is a multiple of the subcarrier spacing $(\Delta f)$, the orthogonality is satisfied not only between subcarriers inside a band, but also between subcarriers from different bands [29]. The inter-band interference is avoided through the orthogonality of each band. Consequently, $\Delta f_{G}$ can be set equal to $\Delta f$ where no frequency guard band is necessary. Multiple OFDM bands can be generated through a multi-carrier optical transmitter and

批注 [gyzhang12]: Reviewer2/com ment4. 2) Page 11 line 57, 'These two schemes are described as single-band OFDM and multi-band OFDM, respectively [23]', here since the focus in on optical network, it will be less confusing to use 'single-band O-OFDM' and 'multiband O-OFDM' instead.

[Authors' Response:] Agree. We have modified the expression according to your suggestion. 
band multiplexing. Upon reception, a filter with bandwidth slightly larger than the bandwidth of each band can be used to select the desired band. This concept is also referred to as orthogonal-bandmultiplexed OFDM (OBM-OFDM) [29], subcarrier multiplexing [30], or cross-channel OFDM (XCOFDM) [9]. The band-multiplexed OFDM scheme without enforcing the band orthogonality has also been discussed in [31].

Using multi-band OFDM, high-speed transmission can be achieved without forcing the subcarriers to be run at extremely high rate, and as such, the DAC/ADC requirements for each subcarrier are significantly relaxed.

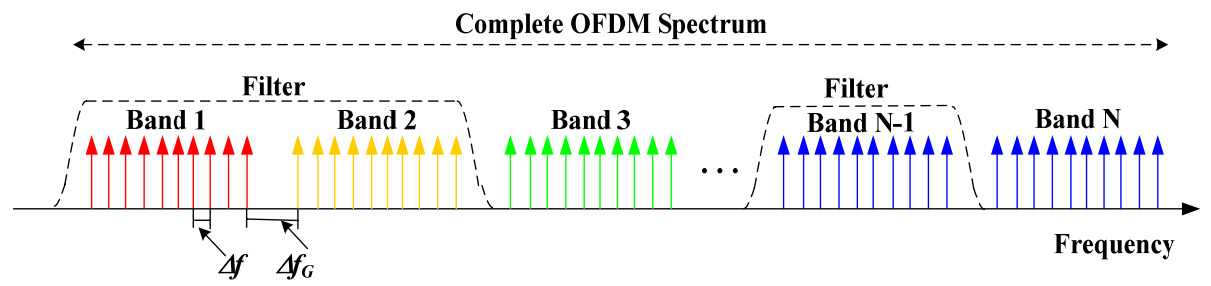

Figure 7 Conceptual diagram of orthogonal-band-multiplexed OFDM (OBM-OFDM) [29].

The FFT-based O-OFDM described in this section has the advantage of simplified optics design. However, this scheme requires guard intervals, training symbols, or pilot carriers, which introduce additional OFDM-specific overhead of around $8 \sim 24 \%$ compared with single-carrier modulation formats (depending on the detailed system design) [32]. To limit the proportion of overhead, a large number of subcarriers $(>>100)$ is normally used in this scheme.

To overcome the large overhead problem of FFT-based O-OFDM, a new Reduced-Guard-Interval (RGI) CO-OFDM scheme was recently introduced [33]. In the RGI-CO-OFDM scheme, a reduced GI between adjacent OFDM symbols is used to accommodate the ISI induced by transmitter bandwidth limitations or fiber polarization mode dispersion (PMD), while fiber chromatic dispersion (CD)induced ISI is compensated at the receiver using electrical dispersion compensation (EDC). As a result, the overhead and OSNR (Optical Signal-to-Noise Ratio) penalty due to the GI are dramatically reduced. Further, a Zero-Guard-Interval (ZGI) CO-OFDM scheme [34] was proposed to completely remove the GI by performing a joint $\mathrm{CD}$ and PMD compensation at the EDC. Comparison of ZGI-COOFDM with RGI-CO-OFDM was reported, showing that ZGI-CO-OFDM demonstrates a superior PMD tolerance than the previous RGI-CO-OFDM scheme, with reasonable small additional computation effort [34].

\subsubsection{Optical Approach}

In the optical approach, an optical OFDM signal is directly generated in the optical domain through modulation of multiple optical subcarriers, without the electrical IFFT processing [35]. The main advantage of the optical approach is that the electronics of the ADC/DAC are eliminated. Different approaches can be used to generate OFDM subcarriers in the optical domain.

\section{(1) All-Optical OFDM}

In the all-optical OFDM scheme [36][37][38][39][40][41], the transmitter generates multiple optical subcarriers from a continuous-wave light source. Each optical subcarrier is then individually modulated, and finally coupled to create an optical OFDM signal, as shown in Figure 8 a). In creating 
the optical OFDM signal, the orthogonal condition is satisfied through proper pulse shaping and phase locking the optical subcarrier to orthogonal frequency, and the baud rate (symbol rate) of each optical subcarrier equals the optical subcarrier spacing.

In its turn, the receiver demultiplexes each optical subcarrier by an all-optical DFT processor, which can be implemented by adding phase delays and careful arrangement of time delays in each subcarrier [36], as depicted in Figure 8b).

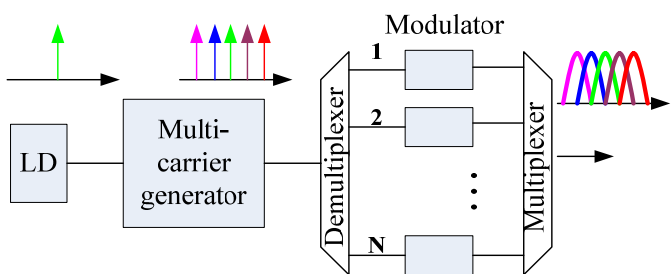

a)

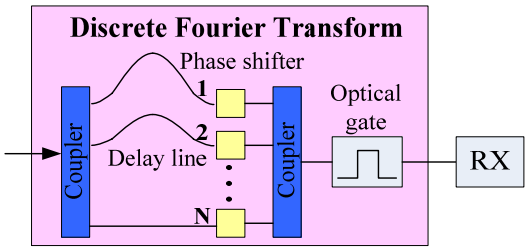

b)

Figure 8 Schematic diagram of the all-optical OFDM [36][42]:

a) Transmitter configuration; b) Receiver configuration.

\section{(2) PDM No-Guard Interval (NGI) CO-OFDM with DSP}

In the all-optical OFDM scheme, normally a low number $(<100)$ of optical subcarriers are preferable, as it corresponds to low numbers of transmitters and receivers and thus minimizes cost and complexity. With a small number of subcarriers, the use of guard interval and training symbols to compensate a CD and PMD-induced distortion will result in excessive overhead or limited compensation capability. Therefore, a No-Guard-Interval (No-GI) coherent OFDM (CO-OFDM) scheme has been proposed, by applying a linear compensation scheme based on digital signal processing (DSP) instead of using GI and training symbols [43][44][45][10][25].

The transmitter configuration of the No-GI CO-OFDM scheme is similar to the optical OFDM scheme described above. At the receiver side, polarization-division multiplexing (PDM) (which will be described in Section3.3) is applied in order to double the spectral efficiency and reduce the operation speed of the ADC and DSP at the receiver side. Furthermore, a DSP is used to equalize the linear distortion with blind adaptive equalizers, and each subcarrier is demultiplexed with a DFT function in the DSP [10], as shown in Figure 9.

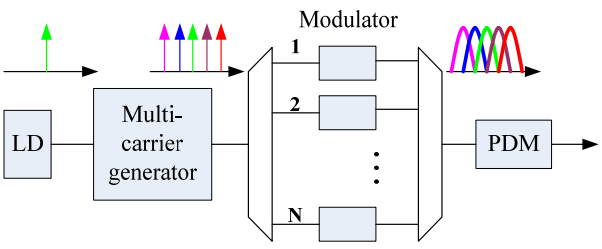

a)

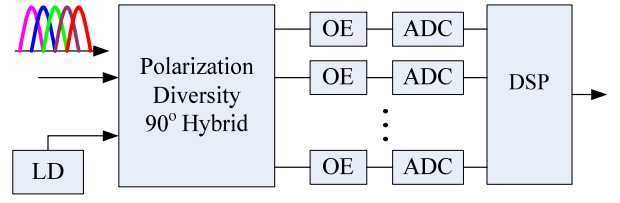

b)

Figure 9 Schematic Diagram of No-GI CO-OFDM:

a) Transmitter configuration; b) Receiver configuration [10].

Table 1 summarizes some of the recent research works related to O-OFDM, classified by the signal synthesis schemes described above. At present, both the electrical and optical approaches of OFDM are advancing quite rapidly, and experiments have shown their high spectrum efficiency and transmission performance. However, for the moment, it is difficult to predict which O-OFDM scheme will dominate 
eventually.

\subsection{O-OFDM Signal Detection Types}

From the signal detection's perspective, optical OFDM can be classified into direct-detection optical OFDM (DDO-OFDM) and coherent-detection optical OFDM (CO-OFDM).

\subsubsection{Direct Detection}

Direct-detection optical OFDM is realized by sending the optical carrier along with the OFDM baseband so that direct detection with a single photodiode can be used at the receiver to convert the optical field back into the electrical domain.

DDO-OFDM can be classified into two categories according to how the optical OFDM signal is generated: (1) linearly-mapped DDO-OFDM, where the optical OFDM spectrum is a linear copy of baseband OFDM [49][50][51], and (2) non-linearly-mapped DDO-OFDM, where the optical OFDM spectrum does not display a replica of baseband OFDM, but aims to obtain a linear mapping between baseband OFDM and optical intensity[52].

The advantage of direct-detection optical OFDM is its relatively-simple implementation and low cost. Therefore, DDO-OFDM has a broader range of applications, such as long-haul transmission [49][53], multi-mode fiber, and short-reach single-mode fiber transmission [54][55]. However, DDOOFDM is less bandwidth efficient, and it has lower OSNR sensitivity compared to CO-OFDM.

\subsubsection{Coherent Detection (CO-OFDM)}

Coherent detection, also referred to as coherent demodulation, is a technique of phase locking to the carrier wave to improve detection. The concept of CO-OFDM was originally proposed in [14]. Recently, more proposals and demonstrations of CO-OFDM have been made [10][29][44][56] [57][58][59][60].

In this approach, a local phase reference or oscillator is mixed with the incoming signal. In this way, the optical analog signals contain all the amplitude, phase, and polarization information before they are received by the photo-detectors and converted into digital streams. Subsequently, the data is recovered by means of DSP where the functions of clock recovery, equalization, carrier phase estimation, and recovery are performed.

When compared to DDO-OFDM, CO-OFDM improves performance in receiver sensitivity, spectral efficiency, and robustness against polarization dispersion [14][44], but it requires higher complexity in transceiver design. The superior performance of CO-OFDM makes it an excellent candidate for longhaul transmission systems, whereas DDO-OFDM is more suitable for cost-effective short-reach applications.

\subsection{MIMO O-OFDM}

In wireless communication systems, the term MIMO (Multiple-Input Multiple-Output) is used to describe a range of systems with multiple transmitting and/or receiving antennas. Depending on the relationship between the signals transmitted from different antennas, MIMO schemes can be used to either increase the overall capacity of the system, or to reduce the probability of outage [59][61]. Because wireless channels usually introduce significant multi-path dispersion, MIMO is often combined with OFDM. 
Table 1 Research works of typical O-OFDM technologies, classified by signal synthesis schemes.

\begin{tabular}{|c|c|c|c|c|c|c|c|c|}
\hline \multicolumn{2}{|c|}{ OFDM types } & \multirow{2}{*}{$\begin{array}{c}\text { Reference } \\
\text { Shieh et al. [14] }\end{array}$} & \multirow{2}{*}{\begin{tabular}{|c|} 
Source \\
Electron. Lett. 2006
\end{tabular}} & \multirow{2}{*}{$\begin{array}{c}\text { Line rate } \\
10 \mathrm{~Gb} / \mathrm{s}\end{array}$} & \multirow{2}{*}{$\begin{array}{c}\begin{array}{c}\# \text { of } \\
\text { subcarriers }\end{array} \\
256\end{array}$} & \multirow{2}{*}{$\begin{array}{c}\text { Modulation format } \\
\text { BPSK }\end{array}$} & \multirow{2}{*}{$\begin{array}{c}\begin{array}{r}\text { Spectrum } \\
\text { efficiency } \\
\text { (b/s/Hz) }\end{array} \\
\text { N/A }\end{array}$} & \multirow{2}{*}{$\begin{array}{c}\begin{array}{c}\text { Distance } \\
\text { (km SSMF) }\end{array} \\
\text { N/A }\end{array}$} \\
\hline FFT- & Single-band & & & & & & & \\
\hline \multirow[t]{9}{*}{ approach } & \multirow{2}{*}{ OFDM } & Shieh et al. [46] & Electron. Lett. 2007 & $10 \mathrm{~Gb} / \mathrm{s}$ & 128 & QPSK & $\mathrm{N} / \mathrm{A}$ & 1000 \\
\hline & & $\begin{array}{l}\text { H. Takahashi et al. } \\
{[47]}\end{array}$ & $\begin{array}{c}\text { J. Lightwave Technol. } \\
2010\end{array}$ & $8 \times 65.1 \mathrm{~Gb} / \mathrm{s}$ & 1024 & PDM-32-QAM & 7 & 240 \\
\hline & \multirow[t]{7}{*}{$\begin{array}{l}\text { Multi-band } \\
\text { OFDM }\end{array}$} & Jansen et al. [26] & $\begin{array}{l}\text { J. Lightwave Technol. } \\
2008\end{array}$ & $25.4 \mathrm{~Gb} / \mathrm{s}$ & 256 & 4-QAM (QPSK) & 2 & 4160 \\
\hline & & Shieh et al. [29] & Opt. Express 2008 & $107 \mathrm{~Gb} / \mathrm{s}$ & $\begin{array}{l}128 / \text { band, } \\
5 \text { bands }\end{array}$ & PDM-QPSK & 2.7 & 1000 \\
\hline & & Jansen et al. [27] [24] & $\begin{array}{c}\text { OFC/NFOEC 2008, J. } \\
\text { Lightwave Technol. } \\
2009\end{array}$ & $10 \times 121.9 \mathrm{~Gb} / \mathrm{s}$ & $\begin{array}{l}\text { 1024/band, } \\
4 \text { bands }\end{array}$ & PDM-QPSK & 2 & 1000 \\
\hline & & Dischler et al. [31] & OFC/NFOEC 2009 & $1.21 \mathrm{~Tb} / \mathrm{s}$ & $\begin{array}{l}340 / \text { band, } \\
10 \text { bands }\end{array}$ & PDM-QPSK & 3.3 & 400 \\
\hline & & Kozicki et al. [28] & $\begin{array}{l}\text { J. Lightwave Technol. } \\
2010\end{array}$ & $1 \mathrm{~Tb} / \mathrm{s}$ & $\begin{array}{l}128 / \text { band, } \\
36 \text { bands }\end{array}$ & PDM-QPSK & 3.3 & 600 \\
\hline & & Liu et al. [33] & $\begin{array}{l}\text { J. Lightwave Technol. } \\
2011\end{array}$ & $448 \mathrm{~Gb} / \mathrm{s}$ & $\begin{array}{l}128 / \text { band } \\
10 \text { bands }\end{array}$ & $\begin{array}{l}\text { PDM-16-QAM, } \\
\text { RGI-CO-OFDM }\end{array}$ & 5.2 & $\begin{array}{c}1600 \\
\text { (ULAF) }\end{array}$ \\
\hline & & Chen et al. [34] & Opt. Express 2011 & $112 \mathrm{~Gb} / \mathrm{s}$ & $\begin{array}{l}128 / \text { band } \\
1 \text { bands }\end{array}$ & $\begin{array}{l}\text { PDM-QPSK, ZGI- } \\
\text { CO-OFDM }\end{array}$ & N/A & $\begin{array}{c}1600 \\
\text { (simulation) }\end{array}$ \\
\hline \multirow{6}{*}{$\begin{array}{l}\text { Optical } \\
\text { approach }\end{array}$} & \multirow{6}{*}{$\begin{array}{l}\text { All-optical } \\
\text { OFDM }\end{array}$} & Sanjoh et al. [36] & OFC/NFOEC 2002 & $15 \mathrm{~Gb} / \mathrm{s}$ & 3 & NRZ & 1 & N/A \\
\hline & & Yonenaga et al. [38] & OFC/NFOEC 2008 & $100 \mathrm{~Gb} / \mathrm{s}$ & 4 & ODB & 1 & 20 \\
\hline & & Lee et al. [37] & Opt. Express 2008 & $100 \mathrm{~Gb} / \mathrm{s}$ & 4 & RZ & 0.625 & 400 \\
\hline & & Hillerkuss et al. [40] & Opt. Express 2010 & $392 \mathrm{~Gb} / \mathrm{s}$ & 9 & DQPSK, DBPSK & N/A & N/A \\
\hline & & Hillerkuss et al. [41] & OFC/NFOEC 2010 & $\begin{array}{c}5.4 \text { and } 10.8 \\
\text { Tbit/s }\end{array}$ & 75 & $\begin{array}{c}\text { PDM-QPSK/ } \\
\text { PDM-16-QAM }\end{array}$ & $2.88 / 5.76$ & $\mathrm{~N} / \mathrm{A}$ \\
\hline & & $\begin{array}{l}\text { D. Hillerkuss et al. } \\
{[39]}\end{array}$ & Nat. Photonics 2011 & $26 \mathrm{~Tb} / \mathrm{s}$ & 325 & 16-QAM & N/A & 50 \\
\hline
\end{tabular}

\section{批注 [gyzhang16]:}

Reviewer1/Comment5a): Page 15:

The caption "Some Research works..." suggests that the survey is merely incomplete. Please make make it complete.

[Authors' Response:] We agree the caption is misleading. Our main objective of this part is to show the typical O-OFDM schemes that are under study in the field, and these contents are also the basis for the enabling technologies of elastic optical network described in Section 4.3.1.1. We have changed the caption to be "Research works of typical O-OFDM technologies, classified by signal synthesis schemes.

批注 [gyzhang17]: Comment 6h) Page 15: If [26] and [23] contain $t$ he same work (looks like from the pa rameters), please collapse the rows. [Authors' Response:] We have merged the two rows. 
A Survey on OFDM-Based Elastic Core Optical Networking

\begin{tabular}{|c|c|c|c|c|c|c|c|}
\hline OFDM types & Reference & Source & Line rate & $\begin{array}{c}\# \text { of } \\
\text { subcarriers }\end{array}$ & Modulation format & $\begin{array}{r}\text { Spectrum } \\
\text { efficiency } \\
\text { (b/s/Hz) }\end{array}$ & $\begin{array}{c}\text { Distance } \\
\text { (km SSMF) }\end{array}$ \\
\hline \multirow{6}{*}{$\begin{array}{l}\text { No-Guard- } \\
\text { Interval } \\
\text { OFDM }\end{array}$} & Kobayashi et al. [25] & OECC 2007 & $110 \mathrm{~Gb} / \mathrm{s}$ & 22 & QPSK & 1 & 80 \\
\hline & Sano et al. [44] & ECOC 2008 & $\begin{array}{c}13.4 \mathrm{~Tb} / \mathrm{s} \\
(134 \times 111 \mathrm{~Gb} / \mathrm{s})\end{array}$ & 2 & PDM-QPSK & 2 & 9612 \\
\hline & Yamada et al. [43] & Electron. Lett. 2008 & $\begin{array}{c}1 \mathrm{~Tb} / \mathrm{s}(10 \times 111 \\
\mathrm{Gb} / \mathrm{s})\end{array}$ & 2 & PDM-QPSK & 2 & 2100 \\
\hline & Yamada et al. [45] & OFC/NFOEC 2008 & $\begin{array}{c}4.1 \mathrm{~Tb} / \mathrm{s}(50 \times 88.8 \\
\mathrm{Gb} / \mathrm{s})\end{array}$ & 2 & PDM-QPSK & 1.65 & 800 \\
\hline & Sano et al. [10] & $\begin{array}{l}\text { J. Lightwave Technol. } \\
2009\end{array}$ & $\begin{array}{c}13.5 \mathrm{~Tb} / \mathrm{s} \\
(135 \times 111 \mathrm{~Gb} / \mathrm{s})\end{array}$ & 2 & PDM-QPSK & 2 & 6248 \\
\hline & $\begin{array}{c}\text { Xia et al. } \\
\quad[48]\end{array}$ & OFC/NFOEC 2011 & $\begin{array}{c}112 \mathrm{~Gb} / \mathrm{s}, 450 \\
\mathrm{~Gb} / \mathrm{s} \text {, and } 1.15 \\
\mathrm{~Tb} / \mathrm{s}\end{array}$ & $2,4,10$ & PDM-QPSK & $\begin{array}{c}3.3 \\
(1.15 \mathrm{~Tb} / \mathrm{s})\end{array}$ & 3560 \\
\hline
\end{tabular}


MIMO, both with and without OFDM, has been successfully applied in single-mode fiber applications by transmitting and receiving signals on both polarizations. In this context, MIMO is also called polarization-division multiplexing (PDM) or dual polarization (DP), whereby data streams are multiplexed on two orthogonal polarization states, thus doubling the total transmission bit rate without increasing the baud rate of the transmission. It has been experimentally shown that, by using MIMO, high-data-rate transmission can be achieved, both in systems using OFDM [35][58][62][63], and in systems using single-carrier formats [59][60][64]

\subsection{Modulation Formats and Adaptive Modulation}

To support high-speed transmission [65], advanced modulation technologies are adopted in OOFDM system to reduce the transmitted symbol rate and achieve higher spectrum efficiency. Multilevel optical modulation, which can encode $m=\log _{2} M$ data bits on $M$ symbols, is an emerging technology for optical high-speed transmission, as the transmission can be accomplished at a symbol rate which is reduced by $m$ compared with the data rate. It allows upgrading to higher data rates under the limits of current high-speed electronics and digital signal processing. On the other hand, with a given data rate, the lower symbol rate supported by multi-level modulation will lead to a drastic reduction of spectrum width. Below, some multi-level modulation formats that are frequently adopted in O-OFDM system are described.

a) M-PSK (M-Phase Shift Keying)

Phase-shift keying (PSK) is a digital modulation scheme that conveys data by modulating the phase of a reference signal (the carrier wave). M-PSK is a multi-level phase modulation technique, where $\mathrm{M}$ is the number of phases used to encode a certain number of bits. Alternatively, instead of using the absolute phase, the phase change of a specified amount can also be used to convey data. Since this scheme depends on the difference between successive phases, it is termed differential phase-shift keying (DPSK).

BPSK (Binary Phase-Shift Keying), also termed 2-PSK, is the simplest form of PSK, where two phases that are separated by $180^{\circ}$ are used, and 1 bit per symbol is supported. QPSK (Quadrature Phase-Shift Keying), also referred to as 4-PSK, uses four phases with $\pi / 2$ phase shifting to represent data, resulting in 2 bits per symbol. DQPSK is the differential QPSK format. Higher-order PSK formats such as 8-PSK can also be employed.

b) M-QAM (M-Quadrature Amplitude Modulation)

M-QAM is a modulation scheme that conveys data by modulating both the amplitude and the phase of a reference signal to increase the bits per symbol. In M-QAM, two M-level amplitudemodulated signals are multiplexed onto two carriers of the same frequency with phase shift of $\pi / 2$. 4QAM, 8-QAM, 16-QAM, 64-QAM, and 256-QAM are defined for 2, 3, 4, 8, and 16 bits/symbol, respectively, as illustrated in Figure 10. 


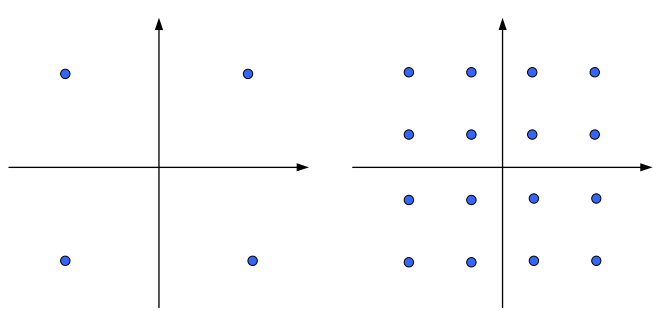

a) 4QAM

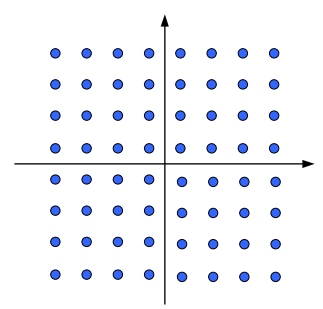

b) 16QAM

c) 64QAM

Figure 10 Example constellation diagrams of QAM modulation.

Amplitude phase-shift keying or asymmetric phase-shift keying (APSK) is also a combination of amplitude modulation and phase modulation schemes. It can be considered as a superclass of QAM. Its advantage over conventional QAM is the lower number of possible amplitude levels, resulting in fewer problems with non-linear amplifiers.

The multi-level optical modulation formats offer high spectral efficiency at the cost of a reduced tolerance to noise according to Shannon's law (which defines the theoretical maximum bit rate that can be transmitted over a bandwidth-limited channel in the presence of noise). Hence, an adaptive modulation technology (also referred to as bit loading [12]) can be adopted to decide what modulation format to use on which subcarrier, based on channel conditions such as reach and signal-to-noise ratio (SNR). The subcarriers with higher SNR can be loaded with higher-level modulation formats in a compact spectrum, while lower SNR subcarriers use lower-level modulation formats in a wider spectrum.

Furthermore, adaptive modulation schemes have the advantage of supporting variable bit rates, which is a desirable feature for future optical networks. Instead of performing changes in hardware, modulation formats can be reconfigured in the DSP and DAC/ADC via software [3][66], ultimately bringing more flexibility to the optical network.

To summarize this section, optical OFDM has received a lot of interest in recent years, leading to a large body of work on various transmission schemes and implementations. Optical OFDM technology has the advantages of superior tolerance to CD/PMD, high spectrum efficiency, and scalability to everincreasing transmission speeds due to its compact subcarrier multiplexing and adaptive modulation scheme. Moreover, it provides capabilities of multiple data rate accommodation and flexible subwavelength bandwidth access. Although real-time O-OFDM implementations are still far from mature due to the lack of high-speed DACs [21], the advantages of O-OFDM show that it is a promising candidate for future optical networks. 


\section{OFDM-Based Elastic Core Optical Network}

\subsection{Elastic Optical Network Concept}

Due to the rapid growth of broadband Internet services and applications such as IPTV, video on demand, and cloud computing, it is expected that diverse bandwidth demands will emerge in future optical networks, with speeds ranging from $\mathrm{Gb} / \mathrm{s}$ up to $\mathrm{Tb} / \mathrm{s}$. Moreover, the temporal as well as the geographical patterns of future Internet traffic will change dynamically.

Although current WDM-based optical network architectures offer advantages of high capacity transmission and reconfigurable wavelength switching, they also present drawbacks of rigid bandwidth and coarse granularity. These may lead to inefficient spectrum utilization and inflexible accommodation of various types of traffic, as each WDM channel occupies the same spectrum width without regard of the transmitted data rate, and each data rate needs a separate transponder which cannot be reconfigured once deployed (although some high-speed (e.g., $100 \mathrm{~Gb} / \mathrm{s}$ ) data-rate-adaptive transponders are currently emerging [66]). Sub-wavelength services could be supported with optical transport network grooming switches; however these electrical switches have high cost and energy consumption. These problems are expected to become even more significant when higher-speed transmission systems (e.g. $100 \mathrm{~Gb} / \mathrm{s}$ and beyond) are deployed.

On the other hand, as transmission speed increases to beyond $100 \mathrm{~Gb} / \mathrm{s}$, e.g., $400 \mathrm{~Gb} / \mathrm{s}$ and $1 \mathrm{~Tb} / \mathrm{s}$, it is not likely for WDM systems to adopt traditional $50 \mathrm{GHz}$ channel spacing for long-haul transmission, because of the increased SNR requirement for higher order-modulation formats, meaning higher rate data will need more spectrum [67]. For example, the bandwidth of a $400 \mathrm{~Gb} / \mathrm{s}$ channel (using PDM 16-QAM with 56-64 Gbaud) is likely to require a $75 \mathrm{GHz}$ channel spacing, while a $1 \mathrm{~Tb} / \mathrm{s}$ channel (using PDM 32-QAM with 112-128Gbaud) would require a $150 \mathrm{GHz}$ channel spacing [67]. Therefore, the optical network needs to support flexible spectrum bandwidth provisioning in order to accommodate future high-speed traffic.

To meet future Internet traffic requirements, a novel elastic optical network architecture with flexible data rate and spectrum allocation, high resource efficiency, low cost, and low power consumption is desirable. Recently, several such network architectures have been proposed, and have drawn increasing attention.

(1) Spectrum-Sliced Elastic Optical Path Network (SLICE)

An OFDM-based elastic optical network architecture was first proposed in [6][70], referred to as SLICE. Using the sub-carrier multiplexing and flexible spectrum allocation features of O-OFDM technology, a bandwidth-elastic optical path can use just enough spectrum (subcarriers) according to the transmitted data rate. As such, by breaking the fixed-grid wavelength-allocation limitation of WDM, it achieves high spectrum efficiency [69]. SLICE supports multiple data rate sub-wavelength or super-wavelength (an optical path that carries traffic at a data rate that is beyond the capacity of a single wavelength/transponder) paths through the introduction of data-rate/bandwidth-variable transponders at the network edge and bandwidth-variable wavelength cross-connects (WXCs) in the network core.

\section{(2) Flexible Optical WDM (FWDM)}

The FWDM network architecture was proposed in [71], and is capable of dynamic allocation of 
network resources, in particular the optical spectrum. It supports optimized spectrum efficiency through elastic channel grids and flexible spectrum allocation for different data rates.

While FWDM has a similar concept of flexible spectrum allocation and data-rate-variable optical path as SLICE, its main difference is that FWDM evolved from the current WDM network architecture, allowing single-carrier modulation as well as OFDM-based multi-carrier modulation schemes. Using single-carrier or multi-carrier modulation schemes may lead to some differences in the data-rate/bandwidth-variable transponder and the transmission system design. Similar to SLICE, network nodes need to be upgraded to spectrum-variable WXCs in the FWDM architecture, and their network planning technologies are equivalent, in general

\section{(3) Data-Rate Elastic Optical Network}

In [72], a data-rate-elastic optical network architecture was proposed, to use a single type of novel rate-tunable transponder which can operate at various data rates to handle all types of traffic. This elastic optical network provides similar solutions as WDM with mixed line rate (MLR) [73], but with increased flexibility, as it simplifies the design of the network and allows sharing of resources for different data-rate services. This elastic optical network architecture can employ multi-carrier and single-carrier multiplexing technologies, while it differs from SLICE and FWDM by using a fixedgrid spectrum allocation. Even though this implies full compatibility with the current WDM network architecture, it fails to achieve flexible spectrum accommodation and high spectrum efficiency. Figure 11 shows the differences in scopes between the three proposed architectures.

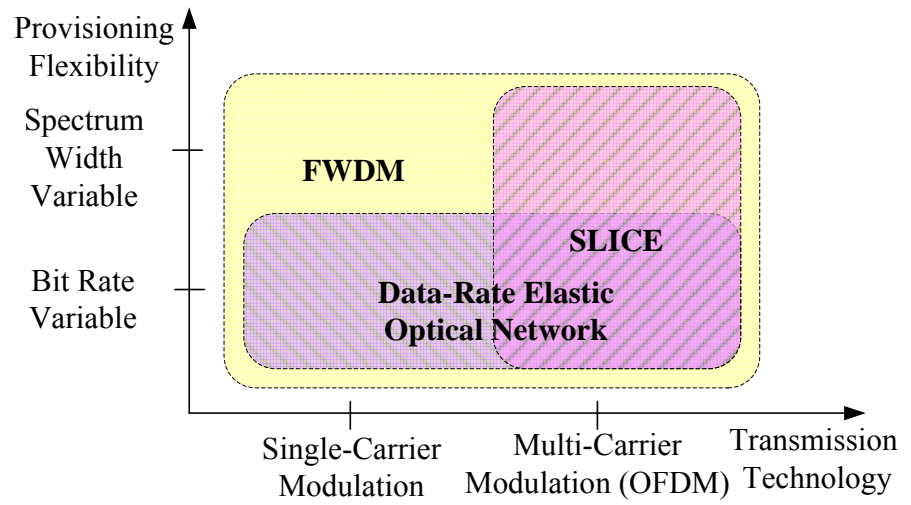

Figure 11 Scopes of SLICE [6], FWDM [71], and Data-Rate Elastic Optical Network [72].

In the following section, we will mainly address the OFDM-based elastic optical network architecture, as the sub-wavelength multiplexing and flexible spectrum allocation features of $\mathrm{O}$ OFDM technology provide immense flexibility to support heterogeneous traffic demands and offer high spectrum efficiency. However, the key enabling technologies can be shared with multi-carrier (OFDM) and single-carrier modulation based elastic optical network, such as data-rate/bandwidthvariable transponder, bandwidth-variable WXC, routing and spectrum allocation schemes, etc. (which will be discussed in detail in Section 4.3).

批注 [gyzhang21]: Reviewer1/Com ment $6 \mathrm{k}$ ) page 18: Not sure about the virtue of Figure 12. Adding the labels of the axes and avoiding the overlap of the shaded fields might help.

Reviewer2/ comment 4.3) Figure 12 in page 20 is not very intuitive because the crossing relationship of the tech terms and complicated layers, can this be modified to show the relationship more clearly? [Authors' Response:] We have modified the figure and added the labels of the axes to make it more. 


\subsection{OFDM-Based Elastic Optical Network Architecture}

In the OFDM-based elastic optical network architecture, multiple data rate sub-wavelength or super-wavelength paths are realized through flexible granular grooming and switching in the spectrum domain, using data-rate/bandwidth-variable transponders and bandwidth-variable WXCs.

The data-rate/bandwidth-variable transponder allocates just enough spectrum (subcarriers) to accommodate sub-wavelength traffic, knows as spectrum slicing [6]. Several OFDM channels can be merged together into a super-channel, transporting a multiple of the capacity of an individual OFDM channel without spectrum guard band in between, as shown in Figure 12(a).

Every WXC node on the optical path allocates a cross-connection with the appropriate spectrum bandwidth to create an appropriate-sized end-to-end optical path. When the traffic increases, the transmitter can increase the transmission capacity, and every WXC on the route expands the switching window, thus enabling data-rate/bandwidth-variable optical paths. Figure 12(b) shows the architecture of the OFDM-based elastic optical network.
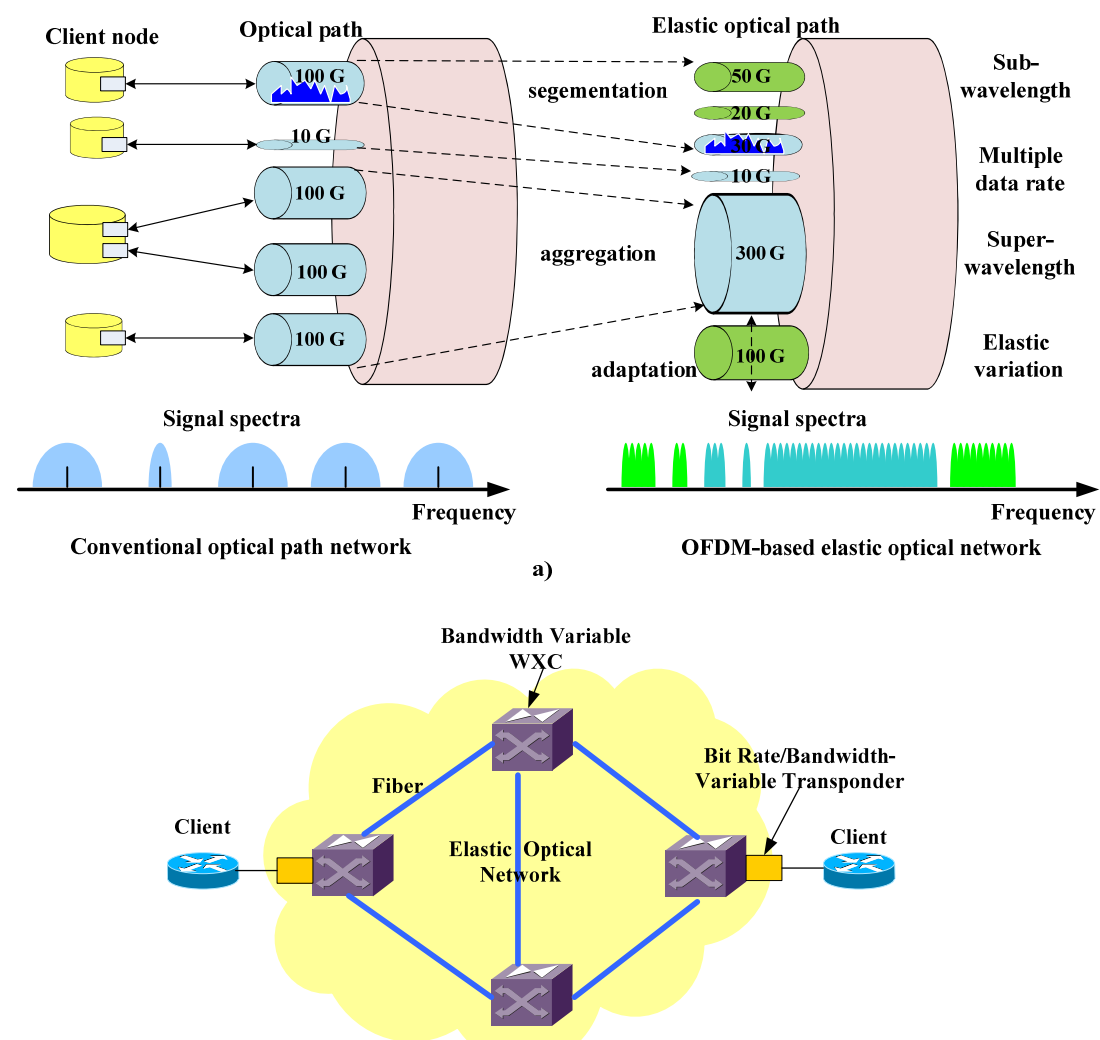

Figure 12 Architecture of OFDM-based elastic optical network [6]: a) Comparison between conventional and elastic optical paths, b) Architecture of elastic optical network.

The benefits of this OFDM-based elastic optical network architecture are as follows.

(1) Supports flexible granularity service aggregation, enabling sub-wavelength, super-wavelength, 
and multiple-rate data traffic accommodation, e.g., $\mathrm{Gb} / \mathrm{s}$ to $\mathrm{Tb} / \mathrm{s}$.

(2) Provides high spectrum efficiency through flexible spectrum allocation according to the transmitted data rate. It has been verified that the spectrum utilization of the elastic optical network improves by 5 95\% compared to a fixed-grid WDM network, although the precise spectrum utilization improvement depends on the network topology and traffic pattern [74].

(3) Supports reach-adaptable line rate, as well as dynamic bandwidth expansion and contraction, through adjusting the number of subcarriers and modulation formats.

(4) Supports energy-efficient operations to save power consumption by turning off some of the OFDM subcarriers when there is not enough traffic for transmission.

(5) Enables optical network virtualization with the virtual link supported by OFDM subcarriers.

As the OFDM-based elastic optical network offers finer bandwidth granularity than WDM and has a coarser granularity than optical packet switching (OPS), it is considered as a middle-term alternative to the as-of-yet immature OPS technology [6].

\subsection{Key Enabling Technologies}

As a new and promising architecture, the OFDM-based elastic optical network has a variety of issues that need to be resolved including re-designing node devices; improving network planning, traffic engineering, and control plane technologies; as well as enhancing current standards.

At the node level, novel data-rate/bandwidth-variable transponders and bandwidth-variable WXCs need to be developed. At the network level, flexible spectrum allocation and routing algorithms, traffic grooming approaches, survivability strategies, network virtualization, energy efficiency, control plane technology need to be studied. Table 2 summarizes the current research in the aforementioned areas.

Table 2 Literature on OFDM-based elastic optical networking.

\begin{tabular}{|c|c|c|}
\hline \multicolumn{2}{|r|}{ Research area } & References \\
\hline \multicolumn{2}{|c|}{ Architecture } & Jinno et al. [6], Jinno et al. [2], Jinno et al. [70]. \\
\hline \multirow[t]{2}{*}{$\begin{array}{l}\text { Node } \\
\text { level }\end{array}$} & $\begin{array}{l}\text { Data-rate/bandwidth-variable } \\
\text { transponder }\end{array}$ & $\begin{array}{l}\text { Jinno et al. [76], Yonenaga et al. [77], Wei et al. [78], } \\
\text { Klekamp et al.[66], Kozicki et al. [79], Takara et al. [80]. }\end{array}$ \\
\hline & $\begin{array}{l}\text { Bandwidth-variable optical } \\
\text { switching and filtering }\end{array}$ & $\begin{array}{l}\text { Jinno et al. [76], Dischler et al. [94], Baxter et al. [89], } \\
\text { Ryf et al. [92], Ryf et al. [91], Frisken et al. [87], } \\
\text { Finisar White Paper [88], Amaya et al. [90], } \\
\text { Kozicki et al. [95]. }\end{array}$ \\
\hline \multirow{7}{*}{$\begin{array}{l}\text { Network } \\
\text { Level }\end{array}$} & Flexible spectrum specification & Jinno et al. [102], ITU-T [98][99]. \\
\hline & $\begin{array}{l}\text { Routing and flexible (non- } \\
\text { uniform) spectrum allocation } \\
\text { algorithm }\end{array}$ & $\begin{array}{l}\text { Zheng et al. [69], Christodoulopoulos et al. [75], } \\
\text { Patel et al. [100], Wang et al. [101], Jinno et al. [102], } \\
\text { Takagi et al. [104], Wan et al. [105], Patel et al. [107], } \\
\text { Takagi et al.[109], Christodoulopoulos et al. [110], } \\
\text { Morea et al.[111], Christodoulopoulos et al. [112]. }\end{array}$ \\
\hline & Traffic grooming & Zhang et al. [114]. \\
\hline & Survivability strategies & Sone et al. [115]. \\
\hline & Network virtualization & $\begin{array}{l}\text { Wei et al. [78], Jinno et al. [117], } \\
\text { Wei et al. [118], Wei et al. [119]. }\end{array}$ \\
\hline & Energy efficiency & Jinno et al. [6]. \\
\hline & Network control and management & Sone et al. [115]. \\
\hline
\end{tabular}

\subsubsection{Node-Level Technologies}

\subsubsection{Data-Rate/Bandwidth-Variable Transponder}

The data-rate/bandwidth-variable transponder is capable of supporting multiple data rates from 
sub-wavelength to super-wavelength. In order to achieve high spectral resource utilization, the datarate/bandwidth-variable transponder needs to generate an optical signal using just enough spectral resources, according to the client data rate and the channel conditions. A variety of schemes can be used to support these features, as described below.

\section{(1) Subcarrier Number Adjustment}

Data rate/bandwidth-variable sub-wavelength services can be provided by adjustment of the number of OFDM subcarriers in the transponder. The control of the subcarrier number can be performed in the optical or digital domain, depending on what kind of signal synthesis method is used (see Section 3.1). The granularity of adjustment corresponds to the data rate of each subcarrier, as illustrated in Figure 13.

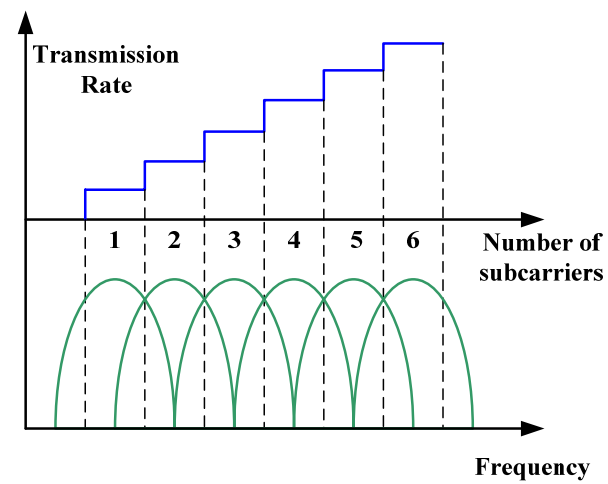

Figure 13 Data-rate/bandwidth variation through subcarrier number adjustment [75].

For O-OFDM systems employing the optical subcarrier synthesis scheme, the data-rate/bandwidthvariable transponder consists of a variable optical multi-carrier source and an optical multi-carrier modulator. The number of subcarriers is controlled by adjusting a continuous-wave light with different oscillator frequencies. In [76], a data-rate/bandwidth-variable transponder that supports bit rates from 40 to $440 \mathrm{~Gb} / \mathrm{s}$, with $10 \mathrm{~Gb} / \mathrm{s}$ granularity and $10 \mathrm{GHz}$ subcarrier spacing was experimentally demonstrated. Likewise, [77] demonstrated an optical transceiver that supports flexible data rates of $107 \mathrm{~Gb} / \mathrm{s}(5 \times 21.4 \mathrm{~Gb} / \mathrm{s}$ DQPSK, $64.2 \mathrm{GHz}), 42.8 \mathrm{~Gb} / \mathrm{s}(2 \times 21.4 \mathrm{~Gb} / \mathrm{s}$ DQPSK, $42.8 \mathrm{GHz}), 32.1 \mathrm{~Gb} / \mathrm{s}$ $(21.4 \mathrm{~Gb} / \mathrm{s}$ DQPSK + 10.7 Gb/s DPSK, 32.1 GHz), and $10.7 \mathrm{~Gb} / \mathrm{s}(1 \times 10.7 \mathrm{~Gb} / \mathrm{s} \mathrm{DPSK}, 21.4 \mathrm{GHz})$. In this scheme, changing the amplitude of the 5 or $10 \mathrm{GHz}$ clocks and adjusting the bias voltage applied to the zero-chirp MZM generates different numbers of subcarriers.

For FFT-based O-OFDM technology, the number of subcarriers can be adjusted in the electrical domain, or the number of bands can be adjusted in the optical domain for the multi-band OFDM scheme. In [78], a $10 \mathrm{~Gb} / \mathrm{s}$ OFDM signal generation with 256 subcarriers was demonstrated, with an adjustment granularity of $40 \mathrm{Mb} / \mathrm{s}$.

\section{(2) Adaptive Modulation}

Using adaptive modulation technology, as described in Section 3.4, a variation of the data rate could be supported by adjusting the modulation format. To increase the transmitted data rate, a higherlevel modulation format with more bits per symbol is chosen, under the limitation of the minimum OSNR required to recover the data, while maintaining a constant symbol rate (which corresponds to a 
constant spectrum width) [66]. This technique has been applied to both single-carrier modulation and multi-carrier modulation schemes [12][72].

Multi-level modulation such as M-QAM, M-PSK, etc. would be good candidates for the adaptive modulation format, and flexible adjustment of the modulation formats could be implemented by software-defined components. For example, the capacity of a subcarrier using one-bit-per-symbol BPSK modulation is $C \mathrm{~Gb} / \mathrm{s}$, and thus QPSK with 2 bits per symbol corresponds to $2 C$, and 16-QAM with 4 bits per symbol corresponds to $4 C$.

Data-rate dynamic adjustment according to the quality of channels was experimentally demonstrated using bit and power loading of optical OFDM subcarriers [12]. Reach and transmission performance versus data-rate variation of OFDM transmission was studied in [66]. A $25 \sim 100 \mathrm{~Gb} / \mathrm{s}$ PDM-QPSK transceiver with modulation-format versatility was demonstrated in [72].

\section{(3) Super-Wavelength Signal Generation}

For super-wavelength services, several OFDM channels can be merged together into a superchannel, transporting a multiple of the capacity of an individual OFDM channel. The data stream is first divided into several channels using layer-2 link aggregation, and then modulated onto continuous OFDM optical paths without guard bands in between. This optically-aggregated super-wavelength path occupies less spectral resources than the corresponding WDM multiplexing method, thereby leaving room for additional traffic.

In [79], an aggregation of seven optical paths into a single spectrally-continuous super-wavelength optical path with a bandwidth of $1 \mathrm{~Tb} / \mathrm{s}$ was achieved. The individual channels are aggregated into one spectrally-continuous super-wavelength optical path, which can be transmitted through the core network consisting of bandwidth-variable wavelength cross-connects (WXCs). In [80], a $420 \mathrm{~Gb} / \mathrm{s}$ distance-adaptive super-channel with DQPSK and 8-APSK modulation was also demonstrated.

A cost comparison between OFDM data-rate variable transponder and 10/40 Gb/s single-carrier transponder was given [82], showing the appropriate cost of the OFDM data-rate variable transponder will lead to total network cost reduction. One of the issues of data-rate/bandwidth-variable transponder technology is that, when it is used to provide transmission for sub-wavelength services, the remaining capacity of the transponder might be wasted. To address this issue, a flxible-bandwidth and format-agile transmitter based on optical arbitrary waveform generation technology [81][83][84] was proposedAQZ

43to provide parallel synthesis of multiple spectral slices inside a transponder, thereby supporting multiple virtual transponders to make full use of the physical transponder. Recently, a multi-flow, multirate, and multi-reach transponder was demonstrated to support flexible point-to-multi-point optical connections [85]. Furthermore, the optical power control of the transponder is a challenging problem, when the number of subcarriers or modulation scheme varies dynamically [12].

Table 3 summarizes recent research work related to data-rate/bandwidth-variable transponders, although we note that research is still ongoing and the appropriate scheme that best fits the requirement of the elastic optical network is an open issue.

Table 3 Research related to data-rate/bandwidth-variable transponder.

\begin{tabular}{|c|c|c|c|}
\hline Reference & Data Rate & Adjustment Granularity & Transmission Scheme \\
\hline Jinno et al. [76] & $40 \sim 440 \mathrm{~Gb} / \mathrm{s}$ & $10 \mathrm{~Gb} / \mathrm{s}$ & No-GI CO-OFDM \\
\hline Yonenaga et al. [77] & $10 \sim 100 \mathrm{~Gb} / \mathrm{s}$ & $\begin{array}{c}21.4 \mathrm{~Gb} / \mathrm{s} \text { (DQPSK) } \\
10.7 \mathrm{~Gb} / \mathrm{s} \text { (DPSK) }\end{array}$ & No-GI CO-OFDM \\
\hline
\end{tabular}


A Survey on OFDM-Based Elastic Core Optical Networking

\begin{tabular}{|c|c|c|c|}
\hline Klekamp et al.[66] & $10 \sim 100 \mathrm{~Gb} / \mathrm{s}$ & $10 \mathrm{~Gb} / \mathrm{s}$ & FFT-based OFDM \\
\hline Wei et al. [78] & $40 \mathrm{Mb} / \mathrm{s} \sim 10 \mathrm{~Gb} / \mathrm{s}$ & $64 \mathrm{Mb} / \mathrm{s}$ & FFT-based OFDM \\
\hline Kozicki et al. [79] & $\begin{array}{c}1 \mathrm{~Tb} / \mathrm{s} \text { super- } \\
\text { wavelength }\end{array}$ & $\begin{array}{c}21.4 \mathrm{~Gb} / \mathrm{s} \text { per subcarrier; } \\
100 \mathrm{~Gb} / \mathrm{s} \text { and } 400 \mathrm{~Gb} / \mathrm{s} \text { per } \\
\text { lightpath }\end{array}$ & No-GI CO-OFDM \\
\hline
\end{tabular}

\subsubsection{Bandwidth-Variable Optical Switching}

To support an end-to-end elastic optical path, every bandwidth-variable WXC (BV-WXC) along the path should allocate an appropriate-sized cross-connection with the corresponding spectrum bandwidth. Therefore, the BV-WXC needs to configure its switching window in a flexible manner according to the spectral width of the incoming optical signal.

The traditional wavelength-selective switch (WSS) is designed for a fixed channel spacing, as there is usually a one-to-one relationship between the WDM channels and switching granularity [86]. In contrast, the novel bandwidth-variable WSS is designed to group more adjacent switching granularities, accommodating a flexible channel width, as shown in Figure 14. Furthermore, finer switching granularity (smaller than the typical channel spacing of $50 \mathrm{GHz}$ or $100 \mathrm{GHz}$ ) is required to fit the subcarrier-level spectrum.

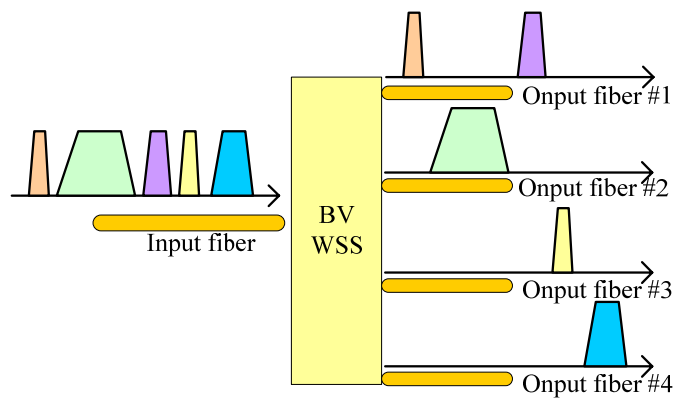

Figure 14 Concept of bandwidth-variable WSS [6]

Liquid crystal or MEMS-based bandwidth-variable WSSs can be employed as switching elements to realize an optical cross-connect with flexible bandwidth and center frequency.

\section{(1) LCoS-Based Bandwidth-Variable WSS}

Liquid Crystal on Silicon (LCoS) is a display technology that combines liquid crystal and semiconductor technologies to create a high-resolution, solid-state display engine. LCoS components are used to control the phase of light at each pixel to produce a programmable grating and beam deflection. The channel bandwidth is software configurable by selecting different numbers of pixels; Refs. [87][88] give detailed descriptions of the bandwidth-flexible WSS employing LCoS technology.

In [89], a BV-WSS based on LCoS was reported with the switching granularity of a multiple of 50 $\mathrm{GHz}$ or $100 \mathrm{GHz}$. In [87], a LCoS-based variable bandwidth filter was implemented, supporting 12.5 $\mathrm{GHz}$ resolution for setting the channel bandwidth. In [90], an experimental demonstration was performed using a LCoS-based flexible spectrum optical switch, supporting $1 \mathrm{GHz}$ resolution with a minimum $12.5 \mathrm{GHz}$ spectrum width.

\section{(2) MEMS-Based Bandwidth-Variable WSS}

A bit-rate and spacing-flexible wavelength blocking filter based on Micro-Electro Mechanical 
System (MEMS) was presented in [91][92]. This blocking filter is based on a free-space diffraction grating, combined with a linear array of single-tilt axes MEMS, as shown in Figure 15. Multiple contiguous $13.2 \mathrm{GHz}$ granularities can be combined to form a wider passband, allowing variation of the spectrum width and the location of the selected channels. Experiments showing the viability of this BV-

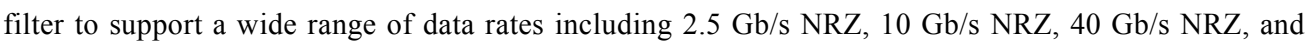
$160 \mathrm{~Gb} / \mathrm{s}$ CSRZ were carried out [91][92].

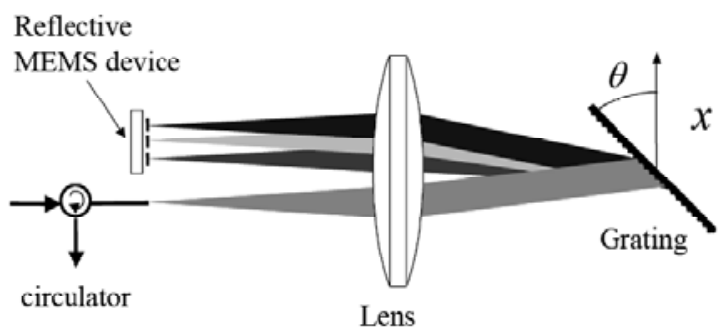

Figure 15 Design of the date-rate and channel-spacing flexible blocking filter [91][92].

\section{(3) Guard Band and Filtering Characteristics}

Theoretically, a continuous-spectrum signal based on OFDM does not have a guard band requirement in the frequency domain between the respective OFDM channels or subbands. However, when an OFDM spectrum signal travels through multiple bandwidth-variable WXCs, the subcarriers on the edge of the spectrum will experience a larger penalty because of the imperfect shape of WSS filters. A guard band between adjacent optical paths can alleviate this problem at the cost of reducing the spectrum efficiency.

Furthermore, if WSS filtering is required to perform add/drop functions in a BV-WXC, some residual signal of adjacent channels besides the added or dropped channels will remain due to the finite slope of the filter edges. To avoid high penalties because of the cross talk, a spectral gap (guard band) must be added between adjacent channels [94].

The guard band size depends on the number of filters (WXC nodes) to cascade, and the filtering characteristics of the WSS. The trade-off between the filter shape and the guard bandwidth was numerically analyzed in [95]. Results show that, for the case of transmission over 10 cascaded filtering stages with second-order Gaussian shape, the optimum guard band for OFDM channels with $10 \mathrm{~Gb} / \mathrm{s}$ subcarriers is $30 \mathrm{GHz}$. Further narrowing of the guard band size could be expected in the future, for instance by increasing the filter order.

\section{(4) Bandwidth-Variable WXC Node Architecture}

A BV-WXC can be built employing bandwidth-variable WSSs mentioned above, using a broadcast-and-select architecture [6][76] or a non-broadcast-and-select architecture [90], as shown in Figure 16. A BV-WXC structure needs to support colorless (any service add/drop to any wavelength), directionless (any service add/drop to any direction), and contentionless (add/drop the same wavelength from different direction) features as described in [68].

In the broadcast-and-select architecture, the input signals are broadcast to all output ports and the appropriate channels are selected on each output port using the bandwidth-variable WSS. In the nonbroadcast-and-select architecture, the input signals are demultiplexed by the bandwidth-flexible WSS, and then routed to different output ports using an optical cross-connect, and finally combined by a coupler at the output port. Optical splitters are used for providing multicast functionality.

A six-node elastic optical network using broadcast-and-select WXCs and liquid crystal-based 
bandwidth-variable WSSs is demonstrated in [76]. The BV-WXC provides variable bandwidth in multiples of $50 \mathrm{GHz}$ to accommodate elastic optical paths, as well as multicast functionality. A $4 \times 4$ LCoS-based WXC with variable channel bandwidth was experimentally demonstrated [96]. The channel bandwidth and center frequency can be tuned with minimum setting resolution of $1 \mathrm{GHz}$. A network experiment using non-broadcast-and-select bandwidth-variable WXC nodes was demonstrated in [90], providing multiple data rate and flexible spectrum switching with $12.5 \mathrm{GHz}$ granularity using LCoS-based WSS

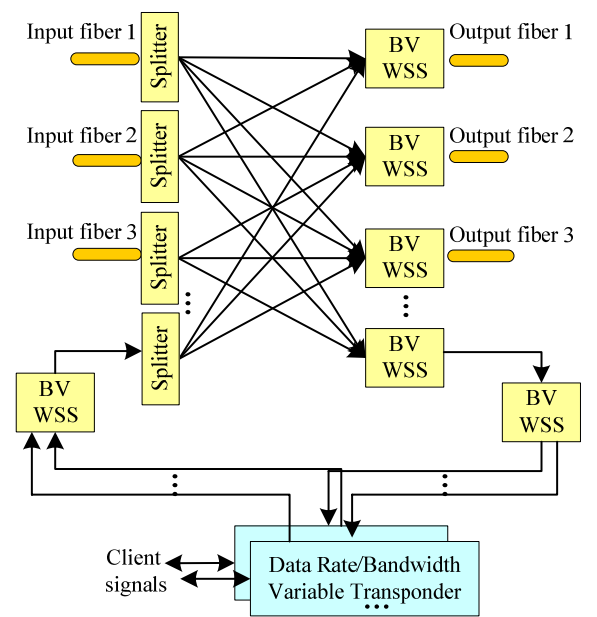

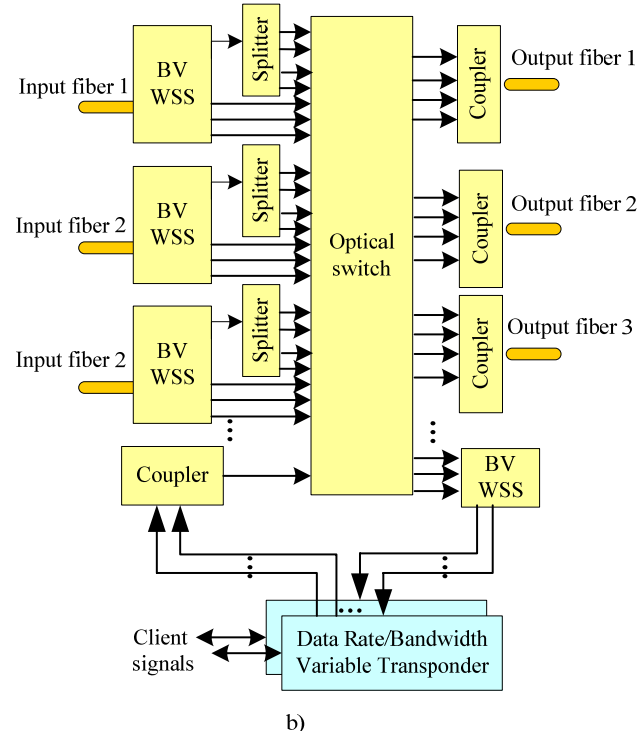

b)

Figure 16 Bandwidth-variable WXC architecture: a) broadcast-and-select architecture [6]; b) non-broadcast-and-select architecture [90].

Table 4 summarizes current research and the capabilities of the bandwidth-variable WSSs.

Table 4 Research related to bandwidth-variable WSS.

\begin{tabular}{|c|c|c|}
\hline Reference & WSS type & Switching/filtering granularity \\
\hline Jinno et al. [76] & LCoS-based WSS & Multiple of 50 GHz \\
\hline Baxter et al. [89] & LCoS-based WSS & Multiple of $50 \mathrm{GHz}$ or $100 \mathrm{GHz}$ \\
\hline Frisken et al. [87] & LCoS-based WSS & Multiple of $12.5 \mathrm{GHz}$ \\
\hline Amaya et al. [90] & LCoS-based WSS & Multiple of $12.5 \mathrm{GHz}$ \\
\hline Ryf et al. [91] [92] & $\begin{array}{c}\text { Free-space diffraction grating } \\
\text { combined with MEMS }\end{array}$ & Multiple of $13.2 \mathrm{GHz}$ \\
\hline
\end{tabular}

As noted in [90][93], the choice of switching granularity is a compromise between operation complexity and flexibility. Standardization work on the elastic channel grid specification is on-going, and will be described in Section 4.3.2.1. The first commercial bandwidth-variable WSS product is now available [88] [86], and will be further improved as the standards move forward.

\subsubsection{Network-Level Technologies}

To enable the elastic optical network, a number of network-level requirements need to be addressed. First, to enforce compliance between diverse implementations, it is important to define a standardized spectrum granularity (grid) specification for flexible spectrum allocation and switching. Second, novel flexible routing and spectrum assignment (RSA) algorithms are required for network 
planning and dynamic resource allocation. Strategies of survivability for the elastic network need to be studied to enable high resiliency against network failures. Other requirements include new network control and management schemes, network virtualization as well as energy efficiency strategies, which are vital to facilitate operation and maintenance of the elastic optical network. In the following sections, these network-level requirements and the related technologies will be discussed.

\subsubsection{Flexible Spectrum Slot Specification}

In current wavelength-routed optical networks, optical channels are aligned to the ITU-T (International Telecommunication Union-Telecommunication Standardization Sector) frequency grid [97]. This rigid and coarsely-granular frequency grid presents a drawback in the form of bandwidth stranding when the volume of end-to-end traffic is not sufficient to fill the entire capacity of a wavelength.

To employ the data-rate-variable and bandwidth-flexible optical network, a flexible spectrum slot specification that could efficiently accommodate sub-wavelength, super-wavelength, and mixed-rate traffic volumes is needed. It should be independent of the signal format and future proof for various new possible data rates. In [102], a potential spectral resource specification was proposed, in which the optical spectrum is divided into a continuous number of frequency slots, and the width of each slot corresponds to the spectrum width of an OFDM subcarrier. There exist two candidates for the flexible spectrum slot specification, as shown in Figure 17.

a) Single Slot on the Grid Approach, which assigns the center frequency and granularity (12.5 $\mathrm{GHz}$ ) of the ITU-T grid to a spectrum slot, and uses the same frequency numbering plan of ITU-T for spectrum slot numbering, thus ensuring compatibility with the current ITU-T grid.

b) Double-Sided Half Slot, which divides one ITU-T frequency grid into two spectral slots $(6.25$ $\mathrm{GHz}$ ), thus introducing a completely new slot numbering system. This scheme provides finer granularity and better flexibility for bandwidth allocation, but without compatibility to the ITU-T standard.

Currently, standardization work related to the flexible grid specification is on-going in ITU-T, where the minimum spectrum slot granularity of $12.5 \mathrm{GHz}$ and central wavelength aligned to 6.25 $\mathrm{GHz}$ frequency step settings are under discussion [98][99].

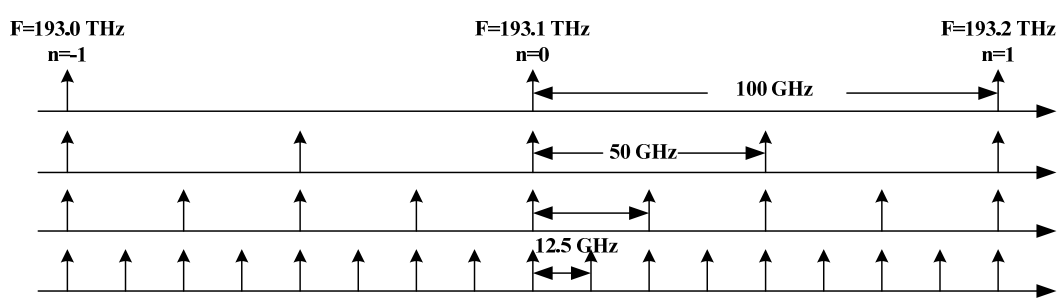

a)

$12.5 \mathrm{GHz}$

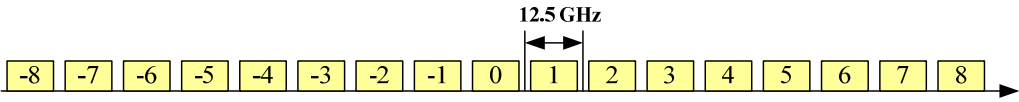

b)

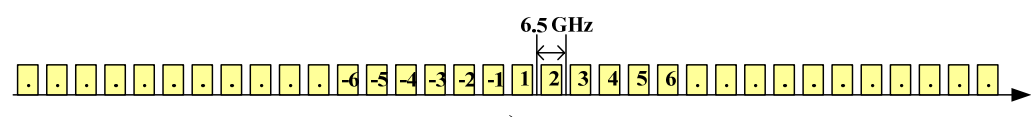

c)

Figure 17 Spectral resource specification schemes: a) current ITU-T WDM frequency grid;

批注 [gyzhang25]: Reviewer2/Com ment 4.5 ) Page 28 line 31, the 'ITU$T$ (International Telecommunication Union-Telecommunication Standardization Sector)' should be introduced in line 5 where it is referred the first time. [Authors' Response:] We have added the definition of ITU-T Page28/line5. 
b) single slot on the grid approach; c) double-sided half slot approach [102].

\subsubsection{Routing and Spectrum Allocation Algorithm}

To establish an elastic optical path that uses a flexible (i.e., non-uniform) spectrum width to accommodate multi-data rate services, the traditional routing and wavelength assignment (RWA) algorithms of WDM networks are no longer directly applicable. New routing and spectrum allocation (RSA) algorithms are needed [6][75].

In RSA, the wavelength-continuity constraint of traditional WDM networks is transformed into a spectrum-continuity constraint. A connection requiring a certain capacity should be satisfied by assigning a number of contiguous subcarrier slots [75]. To route different subcarriers through WXCs and have acceptable signal performance at the receiver side, adjacent OFDM channels need to be separated by spectrum guard bands, each of which normally occupies an integral number of subcarrier spectrum widths (see Section 4.3.1.2 (3)). In addition, the adaptive modulation feature of O-OFDM technology gives the flexibility of choosing modulation formats and adapting the signal spectrum width according to the transmission distance.

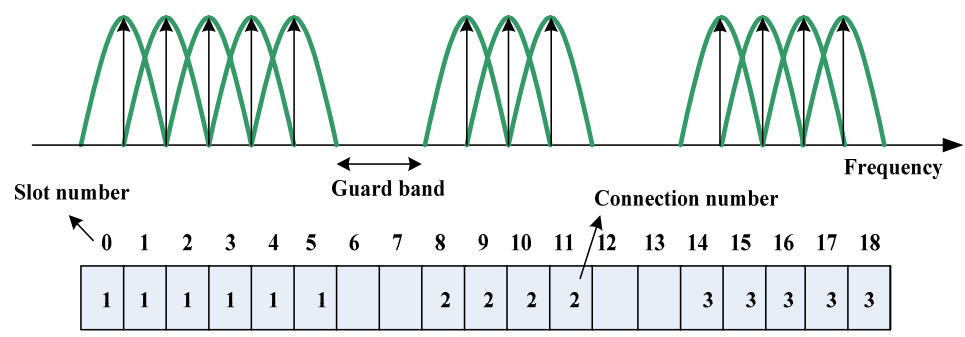

Figure 18 Spectrum allocation as a set of subcarrier slots [75].

Recently, there are an increasing number of research works investigating solutions to the RSA problem of elastic optical networks under both static and dynamic traffic scenarios, including considerations of the distance-adaptive modulation technology. Due to the non-uniform spectrum usage, some spectrum utilization penalty may exist, leaving fragmented spectrum in OFDM links that is difficult to use. Therefore, spectrum allocation policies to minimize fragmentation have been proposed [104], and spectrum defragmentation algorithms to reconfigure the network have been studied [100].

\subsection{Static RSA with ILP (Integer Linear Programing)}

The static solution of RSA deals with the routing and resource allocation during the network planning stage, where an a priori traffic matrix is given in terms of capacity needed, and the routing and spectrum assignment operations are performed off-line. The capacity requirement of a connection is transformed to a number of subcarrier slots (as described in section 4.3.2.1), based on the capacity of each subcarrier.

The static RSA problem has been formulated an ILP that returns the optimum solution through a combined routing and spectrum allocation [75][102]. The objective of the ILP is to minimize the utilized spectrum, with the constraints of spectrum continuity along the path and contiguous subcarrier slots assignment for each connection (no spectrum overlapping among different connections). 
To reduce the complexity of the combined RSA ILP, the RSA problem could be decomposed into (i) the routing and (ii) the spectrum allocation sub-problems, and be addressed separately and sequentially. A sequential ( $\mathrm{R}+\mathrm{SA}$ ) ILP formulation is also given in [75], where the optimum routing was first solved and passed to the spectrum allocation ILP that employs the constraints of continuous spectrum along the route and non-overlapping between different connections. However, the sequential ILP does not guarantee an optimum solution for the RSA problem.

The above ILP formulations are able to find optimum or near-optimum solutions for small networks. However, they are not scalable to large networks, e.g., in a simulation of DT (Deutsche Telekom) network topology with 14 nodes and 46 links, the combined RSA ILP could not return a solution, while the sequential R+SA ILP took several hours [75].

\subsection{Heuristic Algorithms for Static and Dynamic RSA}

To solve the RSA problem efficiently, several heuristic algorithms have been proposed to serve each connection request sequentially in static or dynamic scenarios. The heuristic algorithms can be designed as one-step or two-step approaches.

\section{a) Two-step approach}

The RSA problem can be partitioned into routing and spectrum assignment sub-problems and solved sequentially. Regarding routing, pre-calculated $k$ shortest paths are used in [75][105], and load balanced routing which determines the routing by balancing the load within the network to potentially minimize the spectrum usage in the network is used in [102]. It was shown that shortest-path routing outperforms load-balanced routing in regard to minimizing the total spectrum resources used in the network, while load-balanced routing achieves better performance with the objective to minimize used spectrum index in the network [102].

\section{After routing, spectrum allocation can be done using one of the following policies:}

- First-Fit [104]: In this scheme, all spectrum slots are numbered. With pre-calculated $k$ shortest paths, from the shortest route to the longest one among the candidates, this algorithm searches for the necessary consecutive slots in ascending order of the spectrum slot index, and selects the first found route and slots to the connection request. This algorithm is similar to the first-fit policy for wavelength assignment in WDM networks [106].

- Lowest Starting Slot [75]: For each candidate route, this algorithm searches for the first consecutive slots feasible for the new request in ascending order of the slot index, and selects the path with the lowest starting slot among the set of candidate paths. This algorithm supports void filling, in the sense that voids of size greater than the requested slots can be utilized.

The first-fit policy is quick and simple, while the lowest starting slot allocation might have better spectrum utilization because of its void-filling capability.

To deal with the static request matrix during the network planning stage, the ordering in which the aforementioned single demand heuristic algorithms serve the traffic matrix is of key importance, as different orderings may result in different spectrum utilization. Several ordering policies are proposed in [75]: i) Most-subcarriers-first ordering, which orders the connection demands in decreasing order of

批注 [gyzhang27]: Reviewer1/com ment6 m) Page 30: "However, they are not scalable to large networks, ... hours." should be backed with a reference.

[Authors' Response:] We've added ref[74] to that statement.

批注 [gyzhang28]: [authors :] We have modified the description of the policies to correct some errors in the previous version. 
their requested bandwidth, and serves the connection with the highest bandwidth first; ii) Longestpath-first ordering, which orders connection demands in descending order of the number of links their shortest paths use, and serves the connection that has the longest path first; iii) simulated annealing (SA) meta-heuristic, which finds a near-optimum ordering based on Policies i) and ii) to provide good spectrum allocation solutions. Simulation results indicate that the above heuristics combined using the SA meta-heuristic for ordering can give close-to-optimal solutions in low running times (order of tens of seconds) compared to ILP computation time [75].

\section{b) One-step approach}

Two different algorithms using a one-step approach were proposed in [105], namely Modified Dijkstra's Shortest Path (MSP) and Spectrum-Constraint Path Vector Searching (SCPVS). The two algorithms find the route and the available contiguous spectrum simultaneously. MSP is implemented by checking the available spectrum in the Dijkstra's shortest-path algorithm, and SCPVS builds a path-vector tree with spectrum constraint to search the global optimal route.

Finally, simulation results show that the elastic optical network outperforms the fixed-grid WDM network by less spectrum utilization in the static scenario [74] and lower blocking probability in the dynamic traffic scenario [104][105].

\subsection{RSA for Survivable Networks}

When setting up optical paths in an optical network, it is often desired to set up a link-disjoint or node-disjoint protection path at the same time. An algorithm that provides routing and spectrum allocation for dedicated protection in an elastic optical network with the same spectrum range allocated to both working and protection paths was proposed [107]. Starting at each spectrum slot, an auxiliary graph $G(N, A)$ is established, in which $N$ represents a set of auxiliary nodes and $A$ represents a set of auxiliary links. If there are a number of consecutive available slots that can accommodate the requested connection, an auxiliary link is established on the graph. After constructing the auxiliary graph, two link-disjoint routes can be found using Suurballe's algorithm [108]. By increasing the starting time slot and repeating the above procedure, a number of feasible solutions can be found for each request. The solution with minimum total route length of the working and protection paths is finally selected. A RSA algorithm that deals with dedicated or shared protection in ring networks is provided in [109].

\subsection{Distance-Adaptive RSA}

In traditional WDM networks, all of the optical paths are assigned the same spectrum width, regardless of the transmission distance of each path, thus leading to an inefficient utilization of the spectrum. The distance-adaptive spectrum allocation concept can be introduced to the OFDM-based elastic optical network, based on adaptive modulation and bit-rate/bandwidth variable transponders (as described in Sections 3.4 and 4.3.1.1) to further improve the spectrum efficiency, by adapting a lowlevel modulation format with wider spectrum for longer distance paths and a high-level modulation format with narrower spectrum to shorter distance paths using different modulation formats [102]. For example, for the same data rate, 16-QAM carries twice the number of bits per symbol of QPSK, and consequently requires half the spectrum bandwidth (as shown in Figure 19(a)), while its OSNR tolerance is lower than QPSK, meaning a shorter distance reach. The distance-adaptive spectrum allocation scheme enables spectrum efficiency (SE) improvement, as the assigned spectral bandwidth 
can be saved for shorter paths by increasing the number of modulated bits per symbol.

The distance-adaptive RSA problem is an extension of RSA, with additional requirements to select an appropriate modulation format and spectrum width according to the transmission distance. The distance that a subcarrier with a specific modulation format can reach is determined based on the transport parameters of an optical path, including the transmission distance (OSNR degradation), the number of hops (WXCs that are traversed), and the optical filter shape. Figure 19(b) gives an illustration of the spectral width of different modulation formats vs. optical path reach (number of hops). CD and PMD which can be compensated by the DSP are not considered, and non-linear effects are not taken into account for simplicity. Experimental demonstrations of the distance-adaptive spectrum allocation scheme were carried out, using single-carrier and multi-carrier transmission technologies [103][80].

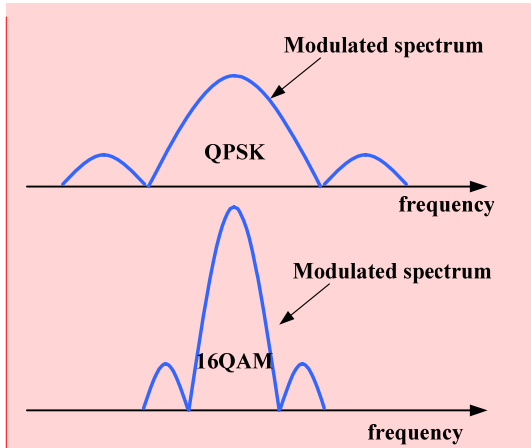

a) Modulated spectrum of QPSK and 16QAM (with the same data rate)

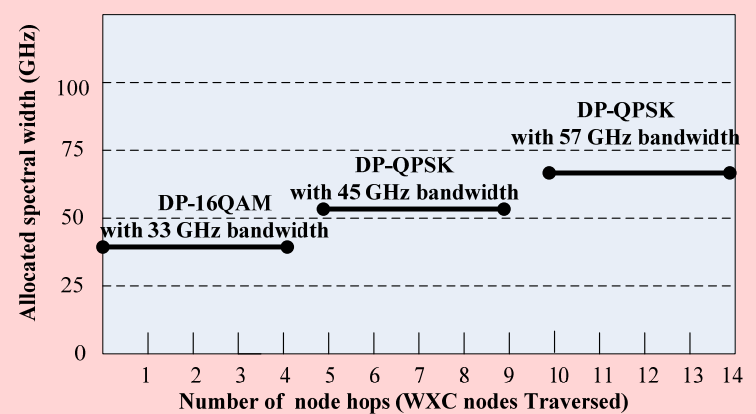

b) Reach vs. allocated spectrum width for different modulation scheme

Figure 19 Distance-adaptive spectrum allocation [102]: a) Modulated spectrum of QPSK and 16-QAM (with the same data rate); b) Reach vs. allocated spectrum width for different modulation scheme (the

spectrum bandwidth listed in the figure includes the necessary guard band. DP-QPSK (Dual Polarization-Quadrature Phase-Shift Keying) signal uses two spectrum widths $45 \mathrm{GHz}$ and $57 \mathrm{GHz}$ because, for a path with more hops, a wider guard band is allocated because of the imperfect WSS filter shape, which has been discussed in Section 4.3.1.2).

A distance-adaptive RSA heuristic algorithm was first proposed in [102], using pre-calculated $k$ shortest path routing and Lowest Starting Slot spectrum allocation scheme. Given the function of transmission distance (number of hops) vs. modulation format, the modulation format and the spectrum width for each subcarrier can be chosen in spectrum allocation stage.

The combined RSA ILP described in Section 4.3.2.2 (1) was extended to give an optimum solution to the distance-adaptive RSA. Additional constraints are added to the ILP formulation, including the reach of each candidate route with the highest modulation level, and the spectrum width needed to be allocated to that route based on the modulation level. A sequential distance-adaptive RSA ILP was proposed in [110], decomposing the problem into the routing and modulation-level-selection ILP, and the subsequent spectrum assignment ILP. Efficient heuristic algorithms were also investigated, incorporating similar spectrum-allocation policies such as Lowest Starting Slot and Void Filling, as described earlier [110].

It has been verified that, with the distance-adaptive spectrum allocation, the allocated spectrum resource reduced by $9 \sim 60 \%$ compared with non-distance-adaptive elastic optical network (spectrum

批注 [gyzhang29]: Reviewer1/Com ment6 n) Page 32: Specify what kind of hops are meant in Figure $20 b$.

[Authors' Response:] We've added an explaination of hops: "number of WXC nodes traversed". 
saving depends on the network topology, traffic pattern, and distance vs. spectrum width of different modulation formats) [109][104].Moreover, the actual spectral gains of the elastic optical network also depend on the guard-band values, transparent/opaque routing constraints, etc. as described in [111].

\subsection{RSA for Time-Varying Traffic}

The data-rate/bandwidth-variable concept of OFDM-based networks brings immense flexibility by supporting time-varying traffic with a transmission rate that fluctuates over time [75]. Network planning of the time-varying traffic models can be done by spectrum reservation and resource sharing among different connections. For example, connections that have complementary transmission rates in time (i.e., when the rate of a connection increases, the opposite tends to happen for another connection), could be served by shared spectrum slots. An alternative approach is to assign each connection a guaranteed transmission rate, and a probabilistic model for exceeding this rate, where spectrum reservation and sharing between connections could be performed [75].

A general policy to allocate subcarriers to time-varying dynamic traffic is proposed in [112]. Two types of subcarriers are defined for a connection, one type is allocated subcarriers for the connection to provide guaranteed service, the other type is reserved subcarriers that could be allocated whenever the service traffic exceeds the allocated capacity, and will be released when traffic decreases. A dynamic algorithm to allocate additional subcarriers to an existing connection was given, and numerical studies are performed to determine the appropriate amount of subcarriers to be reserved.

\subsection{Network Defragmentation RSA}

Under a dynamic traffic scenario, connection setup and release may lead to fragmentation throughout the network, by separating the available spectrum into small non-contiguous spectrum bands. This problem will increase the blocking probability of incoming connection requests, as there may be insufficient contiguous spectrum available. Moreover, because network traffic evolves over time, the allocated optical routes and spectrum might not be optimal in terms of spectral utilization. Therefore, it is desirable for network operators to periodically reconfigure the optical paths and spectrum; this is referred to as network defragmentation.

Unlike the defragmentation operation in SONET/SDH networks, where each link is defragmented independently [113], defragmentation in an elastic optical network requires the optical path to be reconfigured as a whole.

Since defragmentation is performed in a live network, the key requirement is that it should have no (i.e., hitless) impact on existing services. Normally, bridge-and-roll operations, where an alternative connection is first setup and used to carry a copy of the signal (bridge phase) after which the original connection is broken down (roll phase) are performed during defragmentation to guarantee that the service disruption time remains below a certain threshold (e.g., $50 \mathrm{~ms}$ in SONET/SDH). The defragmentation algorithm should also give a step-by-step transition sequence to migrate the connections and minimize the number of moved connections to reduce service disruption [113]. Also, further research is necessary to explore the conditions of when to trigger the defragmentation operation, incorporating metrics such as blocking rate, resource utilization, network capacity, etc.

An ILP formulation of the defragmentation problem of FWDM networks was proposed in [100] 
to provide the optimal defragmentation with considerations of the spectrum continuity and the nonoverlapping spectrum constraints. Two heuristic algorithms, namely, the Greedy-Defragmentation algorithm and the Shortest-Path-Defragmentation algorithm were also provided for large-scale networks, with the objective of maximizing the consolidation of the spectrum as well as minimizing the number of service interruptions. Table 5 summarizes current research on RSA in elastic optical networks. Although considerable progress has been made in this area, we point out that more research is needed on dynamic RSA problems, especially to deal with time-varying traffic models to exploit the data-rate and bandwidth flexibility offered by elastic optical networks.

Table 5 Research on RSA in elastic optical networks.

\begin{tabular}{|c|l|}
\hline RSA algorithms & \multicolumn{1}{|c|}{ Reference } \\
\hline Static RSA & $\begin{array}{l}\text { Zheng et al. [69], Christodoulopoulos et al. [75], } \\
\text { Wang et al. [101] }\end{array}$ \\
\hline Dynamic RSA & Takagi et al. [104], Wan et al. [105] \\
\hline Distance-Adaptive RSA & $\begin{array}{l}\text { Jinno et al. [102], Takagi et al. [104], } \\
\text { Christodoulopoulos et al. [110], Morea et al. [111] }\end{array}$ \\
\hline Time-Varying RSA & Christodoulopoulos et al. [112] \\
\hline Survivable RSA & Patel et al. [107], Takagi et al.[109] \\
\hline Defragmentation RSA & Patel et al. [100] \\
\hline
\end{tabular}

\subsubsection{Traffic Grooming}

In traditional optical WDM networks, traffic grooming is a common functionality, in which multiple low-speed traffic requests are groomed onto a high-capacity lightpath. The technique plays an important role in optimizing resource utilization in WDM networks.

In elastic optical networks, sub-wavelength services could be supported directly by datarate/bandwidth variable transponders and elastic optical paths. However, in this scenario, the capacity of the transponder may not be fully utilized, and the spectral guard bands between the sub-wavelength optical paths may generate a significant amount of overhead.

To address these problems, a traffic-grooming approach in the OFDM-based elastic optical networks was proposed in [114], in which multiple low-speed traffic requests are groomed into elastic optical paths using electrical layer multiplexing. A MILP (Mixed Integer Linear Program) formulation was proposed to minimize the average spectrum utilization in the traffic-grooming scenario. Spectrum savings of $8 \sim 24 \%$ (depending on the guard band width) were demonstrated by the traffic-grooming approach vs. the non-traffic-grooming scenario.

However, as the electrical traffic-grooming approach adds additional OEO conversions and cost to the elastic optical network, more research is needed to address this issue.

\subsubsection{Survivability Strategies}

Survivability is an important aspect of an elastic optical network. By introducing dynamic datarate/bandwidth variation of an elastic optical path, a novel cost-efficient bandwidth squeezing recovery scheme has been proposed [115]. In this scheme, the elastic optical path can be established with a SLA (Service Level Agreement)-based policy, detailing the committed average and peak transmission rates of the client service [116]. In the event of a failure, the proposed scheme can squeeze the traffic that is outside the committed service rate, utilizing the data-rate/bandwidthvariation functionality, when there is not enough bandwidth on the restoration route (see Figure 20). It

批注 [gyzhang32]: Reviewer 4.4) Page 34 line 34, 'SLA-based', here has ' SLA-based' has been introduced before in this paper, otherwise please define it first. [Authors' Response:] We have added the definition of SLA and the related acronym. 
has been demonstrated that this bandwidth-squeezing restoration provides more opportunities to sustain the connectivity in the event of failure than what current rigid optical path networks can provide [115].

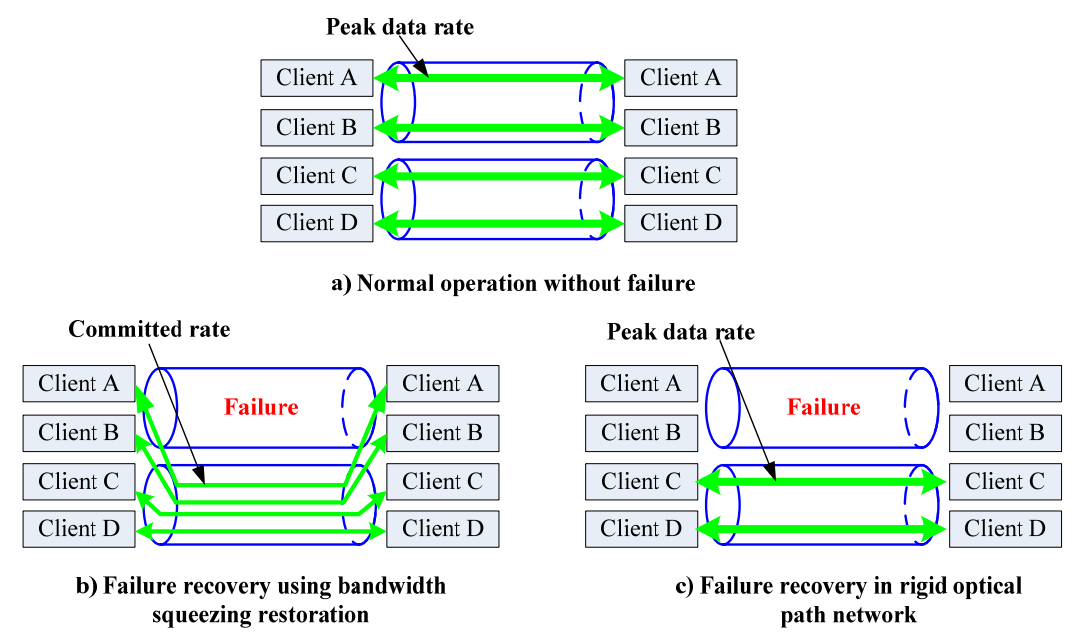

Figure 20 Survivable restoration scheme in elastic optical networks [115].

\subsubsection{Optical Network Virtualization}

For typical applications of network virtualization such as cloud computing and data center interconnections, it is expected that the underlying network provides dynamic and bandwidth-variable connections between geographically-distributed locations [117]. Moreover, for IP-over-optical network configurations, traffic loads may change frequently over time, making it desirable to adapt the virtual topology and virtual link capacity provided by the optical network accordingly [118].

Although current optical networks provide transparency and dynamic wavelength switching, they cannot meet the needs of network virtualization because of their rigid and large bandwidth granularity. OFDM-based elastic optical architecture has great potential to realize optical network virtualization through dynamic bandwidth provisioning with its flexible granularity and bandwidth-tunable capability.

A high-level architecture of a virtualized optical network (VON) was proposed in [117]. The concept of VON is to provide more agility and flexibility to cloud computing environments by adopting the elastic optical path based on O-OFDM technologies and automatically providing any-toany connectivity with the appropriate optical bandwidth.

A programmable and adaptive IP/optical OFDM network architecture was proposed in [78][118]. In this architecture, virtual links are established between router pairs, and are isolated from each other by using different OFDM subcarriers on each physical link. A router interface design using OFDMbased link virtualization techniques is presented in [119], where each router interface contains multiple virtual interfaces that correspond to virtual OFDM links (e.g., M subsets of subcarriers), and the virtual interfaces maintain separate FIFO buffers for each virtual link to store packets. The bandwidth of these virtual links can be changed adaptively by adjusting the modulation format or the number of subcarriers, according to traffic-flow variations. Adjustment decisions are based on 
periodic traffic measurements of the virtual links by backbone routers.

Subcarrier resource assignment for such an IP/optical OFDM network was proposed in [78][118]. Performance comparisons show that a saving of up to $30 \%$ of receivers can be achieved by the adaptive IP/optical OFDM network in comparison to an IP-over-TDM/WDM network [78].

\subsubsection{Energy Efficiency}

Energy consumption and efficiency are becoming major concerns for network operators, as it is of significant importance to build communication networks that enables future energy savings to relax the issues related to the climate change, as well as to scale network architectures supporting rapid growth of traffic demands [120]. The elastic nature of an OFDM-based network provides efficient packing of user traffic demands, and ensures high resource utilization on each OFDM link. This helps to reduce the energy consumption in the network, as less transponders and amplifiers are used compared with a traditional WDM network.

Moreover, in a dynamic traffic scenario where the network load is changing with time, the capacity of an elastic optical path supporting the traffic can change dynamically according to the load, by adjusting the number of subcarriers. Therefore, the data-rate/bandwidth variable transponder can support energy-efficient operations by turning on/off different subcarriers, and adapting its energy consumption according to the amount of traffic to be transported. Also, the optical pump power of the optical amplifiers on the route could be increased or reduced to the appropriate level for supporting the client traffic. These operations will reduce the overall energy consumption of the network [6].

Numerical studies on the energy efficiency of an OFDM-based elastic optical network are expected, and more research work is needed on the design of bit-rate/bandwidth-variable transponders and RSA schemes to support the energy-efficient operations.

\subsubsection{Network Control and Management Scheme}

The OFDM-based elastic network provides immense flexibility in dynamic bandwidth provisioning. Advanced network control and management mechanisms are essential to support the connection provisioning, bandwidth adjustment, and recovery operations in the novel network architecture. The control plane signaling and routing protocols need to be extended to support the spectrum slot specification (see Section 4.3.2.1), distribution of the available spectrum information, bandwidth adjustment, recovery operations, etc. Furthermore, to support the bandwidth-squeezed restoration scheme described in Section 4.3.2.4, two levels of LSP (Label-Switched Path) should be supported by the control plane, to control both OFDM channels and OFDM subcarriers [115].

However, control mechanism to support the dynamic bandwidth variation and related control plane protocol extensions are still unresolved and will be important issues for future research and standardization.

\section{Conclusion}

The rapid growth of Internet traffic and emerging applications are key drivers for high-capacity and cost-effective optical fiber transmission technologies, and they also call for a more data-rateflexible, agile, reconfigurable, and resource-efficient and energy-efficient optical network architecture for the future. Optical OFDM is a promising technology for future high-speed transmission because of its superior tolerance to $\mathrm{CD} / \mathrm{PMD}$, high spectrum efficiency, and scalability to ever-increasing data 
rates based on its subcarrier multiplexing technology. Based on O-OFDM technology, a novel elastic optical network architecture with immense flexibility and scalability on spectrum allocation and data rate accommodation has opened up a new prospect to build a highly-efficient and elastic optical network for the future.

In recent years, a significant amount of research on optical OFDM transmission and elastic optical networking technologies has been reported. In this work, we presented a comprehensive survey that covers optical OFDM transmission and OFDM-based elastic optical networking. OFDM principles including its mathematical formulation, the main building blocks, and related key functions were introduced. To give a general view of the different OFDM implementations for optical transmission, we classified and explained the O-OFDM technologies according to the signal synthesis and detection mechanisms, respectively. The novel elastic optical network architecture based on OFDM as well as the key enabling technologies were discussed, including the data-rate/bandwidth-variable transponder and WXC design at the node level, and routing and spectrum assignment (RSA), traffic grooming, network survivability, virtualization, network control and management solutions at the network level.

As a novel technology and architecture for the future, OFDM-based elastic optical networks pose new challenges on optical transmission system design, flexible spectrum switching node design, network planning, and traffic engineering. Although a lot of effort has been made to solve these problems, there are still many remaining issues, especially on data-rate/bandwidth-variable transponders and WXCs, dynamic routing and spectrum allocation algorithms, network virtualization, energy savings, network control mechanisms, etc. More research is needed to realize the full potential of this novel network architecture.

\section{References}

[1] A. A. M. Saleh and J. M. Simmons, "Technology and Architecture to Enable the Explosive Growth of the Internet," IEEE Communications Magazine, vol. 49, no. 1, pp.126-132, January 2011.

[2] M. Jinno, H. Takara, and B. Kozicki, "Dynamic optical mesh networks: drivers, challenges and solutions for the future," Proc., ECOC2009, Paper 7.7.4.

[3] W. Shieh and I. Djordjevic, “OFDM for Optical Communications,” Academic Press, Elsevier, 2010.

[4] K. Sato and H. Hasegawa, "Optical Networking Technologies That Will Create Future BandwidthAbundant Networks [Invited]," Journal of Optical Communications and Networking, vol. 1, no. 2, pp. 81-93, July 2009.

[5] R. S. Tucker, "Optical packet switching: A reality check," Optical Switching and Networking, vol. 5, no. 1, pp. 2-9, March 2008.

[6] M. Jinno, H. Takara, B. Kozicki, Y. Tsukishima, Y. Sone, and S. Matsuoka, "Spectrum-Efficient and Scalable Elastic Optical Path Network: Architecture, Benefits, and Enabling Technologies," IEEE Communications Magazine, vol. 47, no. 11, pp. 66-73, November 2009.

[7] A. B. Narasimhamurthy, M. K. Banavar, and C. Tepedelenlioglu, “OFDM Systems for Wireless Communications," Morgan \& Claypool Publishers, 2010

[8] S. B. Weinsten and P. M. Ebert, "Data transmission by frequency-division multiplexing using the discrete Fourier transform," IEEE Transactions on Communications, vol. 19, no. 5, pp. 628-634, October 1971 . 
[9] W. Shieh, H. Bao, and Y. Tang, "Coherent optical OFDM: theory and design," Optics Express, vol. 16, no. 2, pp. 841-859, 2008.

[10] A. Sano, E. Yamada, H. Masuda, E. Yamazaki, T. Kobayashi, E. Yoshida,Y. Miyamoto, R. Kudo, K. Ishihara, and Y. Takatori, "No-Guard-Interval Coherent Optical OFDM for 100-Gb/s Long-Haul WDM Transmission,” IEEE/OSA Journal of Lightwave Technology, vol. 27, no. 16, pp. 3705-3713, August 2009.

[11] M. K. Ozdemir and H. Arslan, "Channel estimation for wireless OFDM systems," IEEE Communications Surveys \& Tutorials, vol. 9, no. 2, pp. 18-48, Second Quarter 2007.

[12] Q. Yang, W. Shieh, and Y. Ma, "Bit and Power Loading for Coherent Optical OFDM," IEEE Photonics Technology Letters, vol. 20, no. 15, pp. 1305-1307, August 2008.

[13] A. J. Lowery and J. Armstrong, "Orthogonal-frequency-division multiplexing for dispersion compensation of long-haul optical systems," Optics Express, vol. 14, no. 6, pp. 2079-2084, March 2006.

[14] W. Shieh and C. Athaudage, "Coherent optical orthogonal frequency division multiplexing," Electronics Letters, vol. 42, no. 10, pp. 587- 589, May 2006.

[15] J. M. Tang, P. M. Lane, and K. A. Shore, "Transmission Performance of Adaptively Modulated Optical OFDM Signals in Multimode Fiber Links,” IEEE Photonics Technology Letters, vol. 18, no. 1, pp. 205-207, January, 2006.

[16] J. Lee, F. Breyer, S. Randel, J. Zeng, F. Huijskens, H. P. van den Boom, A. M. Koonen and N. Hanik, "24-Gb/s transmission over 730 m of multimode fiber by direct modulation of 850-nm VCSEL using discrete multi-tone modulation,” Proc., OFC/NFOEC, 2007, Paper PDP6.

[17] S. C. J. Lee, F. Breyer, S. Randel, O. Ziemann, H. P. A. van den Boom, and A. M. J. Koonen, "Low-Cost and Robust 1-Gbit/s Plastic Optical Fiber Link Based on Light-Emitting Diode Technology," Proc., OFC/NFOEC, 2008.

[18] D. Hsu, C. Wei, H. Chen, W. Li, and J. Chen, "Cost-effective 33-Gbps intensity modulation direct detection multi-band OFDM LR-PON system employing a 10-GHz-based transceiver," Optics Express, vol. 19, no. 18, August 2011.

[19] O. Gonzalez, R. Perez-Jimenez, S. Rodriguez, J. Rabadan, and A. Ayala, "Adaptive OFDM system for communications over the indoor wireless optical channel," IEEE ProceedingsOptoelectronics, vol. 153, pp. 139-144, 2006.

[20] J. Grubor, V. Jungnickel, and K. D. Langer, “Adaptive Optical Wireless OFDM System with Controlled Asymmetric Clipping,” Proc., Forty-First Asilomar Conference on Signals, Systems and Computers (ACSSC), pp. 1896-1902, November 2007.

[21] W. Shieh, “OFDM for Flexible High-Speed Optical Networks,” Journal of Lightwave Technology, vol. 29, no. 10, pp. 1560-1577, May 2011.

[22] J. Armstrong, "OFDM for Optical Communications (Invited Tutorial)," IEEE/OSA Journal of Lightwave Technology, vol. 27, no. 3, pp. 525-526, 2009.

[23] V. Chakravarthy, A. S. Nunez, and J. P. Stephens, A. K. Shaw, M. A. Temple, “TDCS, OFDM, and MC-CDMA: A Brief Tutorial,” IEEE Communications Magazine, vol. 43, no. 9, pp. S11-S16, September 2005.

批注 [gyzhang34]: [Authors Comment] Added according to reviewer2's comments. 
[24] S. L. Jansen, I. Morita, T. C. W. Schenk, and H. Tanaka, "121.9-Gb/s PDM-OFDM Transmission With 2-b/s/Hz Spectral Efficiency Over 1000 km of SSMF," Journal of Lightwave Technology, vol. 27 , no. 3, pp. 177-188, February 2009.

[25] T. Kobayashi, A. Sano, E. Yamada, Y. Miyamoto, H. Takara, and A. Takada, "Electro-optically subcarrier multiplexed $110 \mathrm{~Gb} / \mathrm{s}$ OFDM signal transmission over $80 \mathrm{~km}$ SMF without dispersion compensation," Proc., OECC 2007, Paper PD1-6.

[26] S. L. Jansen, I. Morita, T. C. W. Schenk, N. Takeda, and H. Tanaka, "Coherent Optical 25.8-Gb/s OFDM Transmission Over 4160-km SSMF," IEEE/OSA Journal of Lightwave Technology, vol. 26, no. 1, pp. 6-15, January 2008.

[27] S. L. Jansen, I. Morita, and H. Tanaka, “10×121.9-Gb/s PDM-OFDM transmission with $2-\mathrm{b} / \mathrm{s} / \mathrm{Hz}$ spectral efficiency over 1,000 km of SSMF,” Proc., OFC/NFOEC 2008, Paper PDP 2.

[28] Y. Ma, Q. Yang, Y. Tang, S. Chen, and W. Shieh, "1-Tb/s Single-Channel Coherent Optical OFDM Transmission With Orthogonal-Band Multiplexing and Subwavelength Bandwidth Access," IEEE/OSA Journal of Lightwave Technology, vol. 28, no. 4, February 2010.

[29] W. Shieh, Q. Yang, and Y. Ma, "107 Gb/s coherent optical OFDM transmission over 1000-km SSMF using orthogonal band multiplexing," Optics Express, vol. 16, no. 9, pp. 6378-6386, April 2008.

[30] I. B. Djordjevic and B. Vasic, "Orthogonal frequency division multiplexing for high-speed optical transmission,” Optics Express, vol. 14, no. 9, pp. 3767-3775, May 2006.

[31] R. Dischler and F. Buchali, "Transmission of $1.2 \mathrm{~Tb} / \mathrm{s}$ continuous waveband PDM-OFDM-FDM signal with spectral efficiency of $3.3 \mathrm{bit} / \mathrm{s} / \mathrm{Hz}$ over $400 \mathrm{~km}$ of SSMF," Proc., OFC/NFOEC 2009, Paper PDP C2.

[32] S.L. Jansen, I. Morita, T. C. W. Schenk, D. van den Borne, and H. Tanaka, "Optical OFDM - A Candidate for Future Long-Haul Optical Transmission Systems," Proc., OFC/NFOEC 2008, paper OMU3.

[33] X. Liu, S. Chandrasekhar, B. Zhu, P. J. Winzer, A. H. Gnauck, and D. W. Peckham, "448-Gb/s Reduced-Guard-Interval CO-OFDM Transmission Over $2000 \mathrm{~km}$ of Ultra-Large-Area Fiber and Five 80-GHz-Grid ROADMs,” IEEE/OSA Journal of Lightwave Technology, vol. 29, no. 4, February 2011.

[34] C. Chen, Q. Zhuge, and D. V. Plant, "Zero-guard-interval coherent optical OFDM with overlapped frequency-domain CD and PMD equalization,” Optics Express, vol. 19, no. 8, April 2011.

[35] A. Sano, H. Masuda, E. Yoshida, T. Kobayashi, E. Yamada, Y. Miyamoto, F. Inuzuka, Y. Hibino, Y. Takatori, K. Hagimoto, T.Yamada, and Y. Sakamaki, “30×100 Gb/s all-optical OFDM transmission over 1300 km SMF with 10 ROADM nodes,” Proc., ECOC 2007, Paper PD1.7.

[36] H. Sanjoh, E. Yamada, and Y. Yoshikuni, "Optical orthogonal frequency division multiplexing using frequency/time domain filtering for high spectral efficiency up to $1 \mathrm{bit} / \mathrm{s} / \mathrm{Hz}$," Proc., OFC/NFOEC 2002, paper ThD1.

[37] K. Lee, C. T. D. Thai, and J.-K. K. Rhee, “All optical discrete Fourier transform processor for 100 Gbps OFDM transmission,” Optics Express, vol. 16, no. 6, pp. 4023-4028, March 2008.

[38] K. Yonenaga, A. Sano, E. Yamazaki, F.Inuzuka, Y. Miyamoto, A. Takada, and T. Yamada, "100 Gbit/s All-Optical OFDM Transmission Using $4 \times 25$ Gbit/s Optical Duobinary Signals with PhaseControlled Optical Sub-Carriers," Proc., OFC/NFOEC 2008, Paper JThA48.

批注 [gyzhang35]: [Authors Comments] Added according to reviewer2's comments. 
[39] D. Hillerkuss, R. Schmogrow, T. Schellinger, M. Jordan, M. Winter, G. Huber, T. Vallaitis, R. Bonk, P. Kleinow, F. Frey, M. Roeger, S. Koenig, A. Ludwig, A. Marculescu, J. Li, M. Hoh, M. Dreschmann, J. Meyer, S. Ben Ezra, N. Narkiss, B. Nebendahl, F. Parmigiani, P. Petropoulos, B. Resan, A. Oehler, K. Weingarten, T. Ellermeyer, J. Lutz, M. Moeller, M. Huebner1, J. Becker, C. Koos, W. Freude, and J. Leuthold, "26 Tbit s-1 line-rate super-channel transmission utilizing alloptical fast Fourier transform processing,” Nature Photonics, vol. 5, pp. 364-371, June 2011.

[40] D. Hillerkuss, M. Winter, M. Teschke, A. Marculescu, J. Li, G. Sigurdsson, K. Worms, S. Ben Ezra, N. Narkiss, W. Freude, and J. Leuthold, "Simple all-optical FFT scheme enabling Tbit/s realtime signal processing,” Optics Express, vol. 18, no. 9, pp. 9324-9340, April 2010.

[41] D. Hillerkuss, T. Schellinger, R. Schmogrow, M. Winter, T. Vallaitis, R. Bonk, A. Marculescu, J. Li1, M. Dreschmann, J. Meyer, S. Ben Ezra, N. Narkiss, B. Nebendahl, F. Parmigiani, P. Petropoulos, B. Resan, K. Weingarten, T. Ellermeyer, J. Lutz, M. Moller, M. Huebner, J. Becker, C. Koos, W. Freude, and J. Leuthold, "Single Source Optical OFDM Transmitter and Optical FFT Receiver Demonstrated at Line Rates of 5.4 and 10.8 Tbit/s," Proc., OFC/NFOEC 2010, paper PDPC1.

[42] Y. Miyamoto, "Ultra High Capacity Transmission for Optical Transport Network," Proc., OFC/NFOEC 2011, paper OThX4.

[43] E. Yamada, A. Sano, H. Masuda, E. Yamazaki, T. Kobayashi, E.Yoshida, K. Yonenaga, Y. Miyamoto, K. Ishihara, Y. Takatori, T. Yamada, and H. Yamazaki, "1 Tb/s (111 Gbit/s/ch $\times 10 \mathrm{Ch})$ noguard-interval CO-OFDM transmission over $2100 \mathrm{~km}$ DSF,” Electronics Letters, vol. 44, no. 24, pp. 1417-1418, November 2008.

[44] A. Sano, E. Yamada, H. Masuda, E. Yamazaki, T. Kobayashi, E. Yoshida, Y. Miyamoto, S. Matsuoka, R. Kudo, K. Ishihara, Y. Takatori, M. Mizoguchi, K. Okada, K. Hagimoto, H. Yamazaki, S. Kamei, and H. Ishii, "13.4-Tb/s $(134 \times 111-\mathrm{Gb} / \mathrm{s} / \mathrm{ch})$ No-Guard-Interval Coherent OFDM Transmission over 3,600 km of SMF with 19-ps average PMD,” Proc., ECOC 2008, Paper Th.3.E.1.

[45] E. Yamada, A. Sano, H. Masuda, T. Kobayashi, E. Yoshida, Y. Miyamoto, Y. Hibino, K. Ishihara, Y. Takatori, K. Okada, K. Hagimoto, T. Yamada, and H. Yamazaki, "Novel no-guard-interval PDM CO-OFDM transmission in $4.1 \mathrm{~Tb} / \mathrm{s}(55 \times 88.8-\mathrm{Gb} / \mathrm{s})$ DWDM link over $800 \mathrm{~km}$ SMF including 50GHz spaced ROADM nodes,” Proc., OFC/NFOEC 2008, Paper PDP8.

[46] W. Shieh, X. Yi, and Y. Tang, "Transmission experiment of multi-gigabit coherent optical OFDM systems over 1000 km SSMF fiber,” Electronics Letters, vol. 43, pp. 183-184, February 2007.

[47] H. Takahashi, A. A. Amin, S. L. Jansen, I. Morita, and H. Tanaka, "Highly Spectrally Efficient DWDM Transmission at $7.0 \mathrm{~b} / \mathrm{s} / \mathrm{Hz}$ Using $8 \times 65.1-\mathrm{Gb} / \mathrm{s}$ Coherent PDM-OFDM" IEEE/OSA Journal of Lightwave Technology, vol. 28, no. 4, pp. 406-414, February 2010.

[48] T. J. Xia, G. A. Wellbrock, Y.-K. Huang, E. Ip, M. F. Huang, Y. Shao, T. Wang, Y. Aono, T. Tajima, S. Murakami, and M. Cvijetic, "Field Experiment with Mixed Line-Rate Transmission (112$\mathrm{Gb} / \mathrm{s}, 450-\mathrm{Gb} / \mathrm{s}$, and $1.15-\mathrm{Tb} / \mathrm{s}$ ) over 3,560 km of Installed Fiber Using Filterless Coherent Receiver and EDFAs Only," Proc., OFC/NFOEC 2011, Paper PDPA3.

[49] A. J. Lowery, L. Du, and J. Armstrong, "Orthogonal frequency division multiplexing for adaptive dispersion compensation in long haul WDM systems," Proc., OFC/NFOEC 2006, Paper PDP 39.

[50] D. F. Hewitt, "Orthogonal frequency division multiplexing using baseband optical single sideband for simpler adaptive dispersion compensation," Proc., OFC/NFOEC 2007, Paper OME7. 
[51] W. Peng, X. Wu, V. R. Arbab, B. Shamee, J. Y. Yang; L. C. Christen, K. M. Feng, A. E. Willner, and S. Chi, "Experimental demonstration of $340 \mathrm{~km}$ SSMF transmission using a virtual single sideband OFDM signal that employs carrier suppressed and iterative detection techniques," Proc., OFC/NFOEC 2008, Paper OMU1.

[52] M. Schuster, S. Randel, C. A. Bunge, S. C. J. Lee, F. Breyer, B. Spinnler, and K. Petermann, "Spectrally efficient compatible single-sideband modulation for OFDM transmission with direct detection,” IEEE Photonics Technology Letters, vol. 20, no. 9, pp. 670-672, May 2008.

[53] I. B. Djordjevic and B. Vasic, "Orthogonal frequency division multiplexing for high-speed optical transmission,” Optics Express, vol. 14, no. 9, pp. 3767-3775, May 2006.

[54] J. M. Tang and K. A. Shore, "Maximizing the transmission performance of adaptively modulated optical OFDM signals in multimode-fiber links by optimizing analog-to-digital converters," IEEE/OSA Journal of Lightwave Technology, vol. 25, no. 3, pp. 787-798, March 2007.

[55] X. Q. Jin, J. M. Tang, P. S. Spencer, K. A. Shore, "Optimization of adaptively modulated optical OFDM modems for multimode fiber-based local area networks [Invited]," OSA Journal of Optical Networking, vol. 7, no. 3, pp. 198-214, March 2008.

[56] W. Shieh, X. Yi, Y. Ma, Y. Tang, "Theoretical and experimental study on PMD-supported transmission using polarization diversity in coherent optical OFDM systems," Optics Express, vol. 15, no. 16, pp. 9936-9947, August 2007.

[57] W. Shieh, "High Spectral Efficiency Coherent Optical OFDM for $1 \mathrm{~Tb} / \mathrm{s}$ Ethernet Transport," Proc., OFC/NFOEC 2009, Paper OWW1.

[58] Y. Ma, W. Shieh, and Q. Yang, "Bandwidth-efficient $21.4 \mathrm{~Gb} / \mathrm{s}$ coherent optical $2 \times 2 \mathrm{MIMO}$ OFDM transmission," Proc., OFC/NFOEC 2008, Paper JWA59.

[59] A. F. Molisch and M. Z.Win, "MIMO systems with antenna selection," IEEE Microwave Magazine, vol. 5, no. 1, pp. 46-56, March 2004.

[60] G. Charlet, J. Renaudier, H. Mardoyan, O. B. Pardo, F. Cerou, P. Tran, and S. Bigo, "12.8 Tbit/s transmission of 160 PDM-QPSK $(160 \times 2 \times 40 \mathrm{~Gb} / \mathrm{s})$ channel with coherent detection over $2,550 \mathrm{~km}$," Proc., ECOC 2007, Paper PD1.6.

[61] A. J. Paulraj, D. A. Gore, R. U. Nabar, and H. Bolcskei, "An overview of MIMO communications-A key to gigabit wireless,” Proceedings of the IEEE, vol. 92, pp. 198-218, 2004.

[62] W. Shieh, "PMD-supported coherent optical OFDM systems," IEEE Photonics Technology Letters, vol. 19, no. 3, pp. 134-136, February 2007.

[63] S. L. Jansen, I. Morita, and H. Tanaka, " $16 \times 52.5-\mathrm{Gb} / \mathrm{s}, 50-\mathrm{GHz}$ spaced, OLMUX-CO-OFDM transmission over 4,160 km of SSMF enabled by MIMO processing KDDI R\&D Laboratories," Proc., ECOC 2007, Paper PD1.3.

[64] S. Chandrasekhar, X. Liu, E. C. Burrows, and L. L. Buhl, "Hybrid 107-Gb/s polarizationmultiplexed DQPSK and 42.7-Gb/s DQPSK transmission at $1.4-\mathrm{bits} / \mathrm{s} / \mathrm{Hz}$ spectral efficiency over $1280 \mathrm{~km}$ of SSMF and 4 bandwidth-managed ROADMs," Proc., ECOC 2007, Paper PD 1.9.

[65] J. Proakis, "Digital Communications,” McGraw Hill, 2000.

[66] A. Klekamp, O. Rival, A. Morea, R. Dischler, and F. Buchali, "Transparent WDM Network with Bitrate Tunable Optical OFDM Transponders,” Proc., OFC/NFOEC 2010, Paper NTuB5. 
[67] A. Nag, M. Tornatore, and B. Mukherjee, "Optical Network Design With Mixed Line Rates and Multiple Modulation Formats," IEEE/OSA Journal of Lightwave Technology, vol. 28, no. 4, pp. 466475, February 2010.

[68] S. Gringeri, B. Basch, V. Shukla, R. Egorov, and T. J. Xia, "Flexible Architectures for Optical Transport Nodes and Networks," IEEE Communications Magazine, vol. 48, no. 7, pp. 40-50, July 2010

[69] W. Zheng, Y. Jin, W. Sun, W. Guo, and W. Hu, "On the Spectrum-Efficiency of BandwidthVariable Optical OFDM Transport Networks,” Proc., OFC/NFOEC 2010, Paper OWR5.

[70] M. Jinno, H. Takara, and B. Kozicki, "Concept and Enabling Technologies of Spectrum-Sliced Elastic Optical Path Network (SLICE)", Proc., Communications and Photonics Conference and Exhibition (ACP), November 2009, Paper FO2.

[71] A. N. Patel, P. N. Ji, J. P. Jue, and T. Wang, "Routing, Wavelength Assignment, and Spectrum Allocation in Transparent Flexible Optical WDM (FWDM) Networks," Photonics in Switching, OSA Technical Digest 2010, Paper PDPWG1.

[72] O. Rival and A. Morea, "Elastic Optical Networks with 25-100G Format-Versatile WDM Transmission Systems," Proc., OECC2010, Paper 7A2-3.

[73] A. Nag, M. Tornatore, and B. Mukherjee, "Optical Network Design With Mixed Line Rates and Multiple Modulation Formats," IEEE/OSA Journal of Lightwave Technology, vol. 28, no. 4, pp. 466475, February 2010.

[74] K. Christodoulopoulos, I. Tomkos, and E. Varvarigos, "Spectrally/Bitrate Flexible Optical Network Planning,” Proc., ECOC 2010, Paper We.8.D.3.

[75] K. Christodoulopoulos, I. Tomkos, and E. A. Varvarigos, "Routing and Spectrum Allocation in OFDM-based Optical Networks with Elastic Bandwidth Allocation,” Proc., GlobeCom 2010.[76] M. Jinno, H. Takara, B. Kozicki, Y. Tsukishima, T. Yoshimatsu, T. Kobayashi, Y. Miyamoto, K. Yonenaga, A. Takada, O. Ishida, and S. Matsuoka, "Demonstration of Novel Spectrum-Efficient Elastic Optical Path Network with Per-Channel Variable Capacity of $40 \mathrm{~Gb} / \mathrm{s}$ to Over $400 \mathrm{~Gb} / \mathrm{s}$," Proc., ECOC 2008, Paper Th.3.F.6.[77] K. Yonenaga, F. Inuzuka, S. Yamamoto, H. Takara, B. Kozicki, T. Yoshimatsu, A. Takada, and M. Jinno, "Bit-Rate-Flexible All-Optical OFDM Transceiver Using Variable MultiCarrier Source and DQPSK/DPSK Mixed Multiplexing,” Proc., OFC/NFOEC 2009, Paper OWM1.

[78] W. Wei, J. Hu, D. Qian, P. N. Ji, T. Wang, X. Liu, and C. Qiao, "PONIARD: A Programmable Optical Networking Infrastructure for Advanced Research and Development of Future Internet," IEEE/OSA Journal of Lightwave Technology, vol. 27, no. 3, pp. 233-242, February 2009.

[79] B. Kozicki, H. Takara, Y. Tsukishima, T. Yoshimatsu, T. Kobayashi, K. Yonenaga, and M. Jinno, "Optical Path Aggregation for 1-Tb/s Transmission in Spectrum-Sliced Elastic Optical Path Network," IEEE Photonics Technology Letters, vol.22, no.17, pp.1315-1317, September 2010.

[80] H. Takara, B. Kozicki, Y. Sone, T. Tanaka, A. Watanabe, A. Hirano, K. Yonenaga, and M. Jinno, "Distance-Adaptive Super-Wavelength Routing in Elastic Optical Path Network (SLICE) with Optical OFDM," Proc., ECOC 2010, Paper We.8.D.2.

[81] T. He, N. K. Fontaine, R. P. Scott, D. J. Geisler, L. Paraschis, O. Gerstel, J. P. Heritage, and S. J. B. Yoo, "Flexible-Bandwidth and Format-Agile Networking Based on Optical Arbitrary Waveform Generation and Wavelength Selective Switches," 23rd Annual Meeting of the IEEE Photonics Society, 
2010, Paper MM6.

[82] A. Bocoi, M. Schuster, F. Rambach, D. A. Schupke, C. Bunge, and B. Spinnler, "Cost Comparison of Networks Using Traditional 10 and $40 \mathrm{~Gb} / \mathrm{s}$ Transponders Versus OFDM Transponders," Proc., OFC/NFOEC 2008, Paper OThB4.

[83] D. J. Geisler, N. K. Fontaine, R. P. Scott, T. He, L. Paraschis, O. Gerstel, J. P. Heritage, and S. J.

B. Yoo, "Bandwidth Scalable, Coherent Transmitter Based on Parallel Synthesis of Multiple Spectral Slices," Proc., OFC/NFOEC 2011, Paper OTuE3.

[84] T. He, R. P. Scott, D. J. Geisler, N. K. Fontaine, O. Gerstel, L. Paraschis, J. P. Heritage and S. J. B. Yoo, "Flexible-Bandwidth, Impairment-Aware Transmitter Based on Parallel Synthesis of Optical Frequency Combs," Proc., OFC/NFOEC 2011, Paper OtuE4.

[85] H. Takara, T. Goh, K. Shibahara, K. Yonenaga, S. Kawai, and M. Jinno, "Experimental Demonstration of $400 \mathrm{~Gb} / \mathrm{s}$ Multi-flow, Multirate, Multi-reach Optical Transmitter for Efficient Elastic Spectral Routing,” Proc. ECOC 2011, Paper Tu.5.A.4.

[86] T. A. Strasser and J. L. Wagener, "Wavelength-Selective Switches for ROADM Applications," IEEE Journal of Selected Topics in Quantum Electronics, vol. 16, no. 5, pp. 1150-1157, September/October 2010.

[87] S. Frisken, G. Baxter, D. Abakoumov, H. Zhou, I. Clarke, and S. Poole, "Flexible and Grid-less Wavelength Selective Switch using LCOS Technology,” Proc., OFC/NFOEC 2011, Paper OTuM3.

[88] "Programmable narrow-band filtering using the WaveShaper 1000E and WaveShaper 4000E," Finisar White Paper.

[89] G. Baxter, S. Frisken, D. Abakoumov, H. Zhou, I. Clarke, A. Bartos and S. Poole, "Highly programmable Wavelength Selective Switch based on Liquid Crystal on Silicon switching elements" Proc., OFC/NFOEC 2006, Paper OTuF2.

[90] N. Amaya, I. Muhammad, G. S. Zervas, R. Nejabati, D. Simeodinou, Y. R. Zhou, and A. Lord, "Experimental Demonstration of a Gridless Multi-granular Optical Network Supporting Flexible Spectrum Switching," Proc., OFC/NFOEC 2011, Paper OMW3.

[91] R. Ryf, Y. Su, L. Möller, S. Chandrasekhar, D. T. Neilson, and C. R. Giles,” Data rate and channel spacing flexible wavelength blocking filter," Proc., OFC/NFOEC 2004, Paper PDP10.

[92] R. Ryf, Y. Su, L.Moller, S. Chandrasekhar, X. Liu, D. T. Neilson, and C. R. Giles, "Wavelength blocking filter with flexible data rates and channel spacing," IEEE/OSA Journal of Lightwave Technology, vol. 23, no. 1, pp. 54-61, January 2005.

[93] G. Shen and Q. Yang, "From Coarse Grid to Mini-Grid to Gridless: How Much can Gridless Help Contentionless?” Proc., OFC/NFOEC 2011, Paper OTuI3.

[94] R. Dischler, F. Buchali, and A. Klekamp, "Demonstration of Bit Rate Variable ROADM functionality on an Optical OFDM Superchannel," Proc., OFC/NFOEC 2010, Paper OTuM7.

[95] B. Kozicki, H. Takara, T. Yoshimatsu, K. Yonenaga, and M. Jinno, "Filtering Characteristics of Highly-Spectrum Efficient Spectrum-Sliced Elastic Optical Path (SLICE) Network," Proc., OFC/NFOEC 2009, Paper JWA43.

[96] Y. Sakurai, M. Kawasugi, Y. Hotta, M. S. Khan, H. Oguri, K. Takeuchi, S. Michihata, and N. Uehara, "LCOS-Based 4x4 Wavelength Cross-Connect Switch For Flexible Channel Management in 
ROADMs," Proc., OFC/NFOEC 2011, Paper OTuM4.

[97] ITU-T G.694.1 “Spectral grids for WDM applications: DWDM frequency grid,” 2002.

[98] ITU-T C1284, "Proposal of subjects to be discussed regarding flexible grids," January 2011.

[99] ITU-T C1288, "Extension of Rec. G.694.1 by a new clause to address flexible frequency grids," January 2011.

[100] A. N. Patel, P. N. Ji, J. P. Jue, and T. Wang, "Defragmentation of Transparent Flexible Optical WDM (FWDM) Networks,” Proc., OFC/NFOEC 2011, Paper OTuI8.

[101] Y. Wang, X. Cao, and Q. Hu, "Routing and Spectrum Allocation in Spectrum-sliced Elastic Optical Path Networks," Proc., International Conference on Communications (ICC), IEEE, June 2011.

[102] M. Jinno, B. Kozicki, H. Takara, A. Watanabe, Y. Sone, T. Tanaka, and A. Hirano, "Distance adaptive spectrum resource allocation in spectrum-sliced elastic optical path network," IEEE Communications Magazine, vol. 48, no. 8, pp. 138-145, August 2010.

[103] B. Kozicki, H. Takara, Y. Sone, A. Watanabe, and M. Jinno, "Distance-Adaptive Spectrum Allocation in Elastic Optical Path Network (SLICE) with Bit per Symbol Adjustment," Proc., OFC/NFOEC 2010, Paper OMU3.

[104] T. Takagi, H. Hasegawa, K. Sato, Y. Sone, B. Kozicki, A. Hirano, and M. Jinno, "Dynamic Routing and Frequency Slot Assignment for Elastic Optical Path Networks that Adopt Distance Adaptive Modulation,” Proc., OFC/NFOEC 2011, Paper OTuI7.

[105] X. Wan, L. Wang, N. Hua, H. Zhang, and X. Zheng, "Dynamic Routing and Spectrum Assignment in Flexible Optical Path Networks,” Proc., OFC/NFOEC 2011, Paper JWA55.

[106] H. Zhang, J. P. Jue, B. Mukherjee, "A Review of Routing and Wavelength Assignment Approaches for Wavelength-Routed Optical WDM Networks," Optical Networks Magazine, vol. 1, no. 1, pp. 47-60, January 2000.

[107] A. N. Patel, P. N. Ji, J. P. Jue, and T. Wang, "Survivable Transparent Flexible Optical WDM (FWDM) Networks," Proc.,OFC/NFOEC 2011, Paper OTuI2.

[108] J. W. Suurballe and R. E. Tarjan, "A quick method for finding shortest pairs of disjoint paths," Networks, vol. 14, no. 2, pp. 325-336, 1984.

[109] T. Takagi, H. Hasegawa, K. Sato, T. Tanaka, B. Kozicki, Y. Sone, and M. Jinno, “Algorithms for Maximizing Spectrum Efficiency in Elastic Optical Path Networks that Adopt Distance Adaptive Modulation," Proc., ECOC 2010, Paper. We.8.D.5.

[110] K. Christodoulopoulos, I. Tomkos, and E. A. Varvarigos, "Elastic Bandwidth Allocation in Flexible OFDM-Based Optical Networks," IEEE/OSA Journal of Lightwave Technology, vol. 29, no. 9, pp. 1354-1366, May 2011.

[111] A. Morea, A. F. Chong, and O. Rival, "Impact of transparent network constraints on capacity gain of elastic channel spacing," Proc., OFC/NFOEC 2011, Paper JWA62.

[112] K. Christodoulopoulos, I. Tomkos and E. Varvarigos, "Dynamic Bandwidth Allocation in Flexible OFDM-based Networks,” Proc., OFC/NFOEC 2011, Paper OTuI5.

[113] S. Acharya, B. Guptu, P. Risbood, and A. Srivastuvu, "Mobipack: optimal hitless SONET defragmentation in near-optimal cost,” Proc., INFOCOM 2004, vol.3, pp. 1819-1829. 
[114] Y. Zhang, X. Zheng, Q. Li, N. Hua, Y. Li, and H. Zhang, "Traffic Grooming in Spectrum-Elastic Optical Path Networks,” Proc., OFC/NFOEC 2011, Paper OTuI1.

[115] Y. Sone, A. Watanabe, W. Imajuku, Y. Tsukishima, B. Kozicki, H. Takara, and M. Jinno, "Highly Survivable Restoration Scheme Employing Optical Bandwidth Squeezing in Spectrum-Sliced Elastic Optical network SLICE," Proc., OFC/NFOEC 2009, Paper OThO2.

[116] Optical Internetworking Forum, "User Network Interface (UNI) 2.0 Signaling Specification," 2008 .

[117] M. Jinno and Y. Tsukishima, "Virtualized Optical Network (VON) for Agile Cloud Computing Environment," Proc., OFC/NFOEC 2009, Paper OMG1.

[118] W. Wei, C Wang, and X. Liu, “Adaptive IP/Optical OFDM Networking Design,” Proc., OFC/NFOEC 2010, Paper OWR6.

[119] W. Wei, J. Hu, C. Wang, T. Wang, and C. Qiao, “A Programmable Router Interface Supporting Link Virtualization with Adaptive Optical OFDMA Transmission,” Proc., OFC/NFOEC 2009, Paper JWA68.

[120] S. Aleksic, "Energy-Efficient Global Networks and Their Implications," Proc., 10th International Conference on Telecommunications (ConTEL), pp. 331-338, June 2009.

\section{Acronyms}

$\begin{array}{ll}\text { ADC } & \text { Analog-to-Digital Converter } \\ \text { APSK } & \text { Amplitude Phase-Shift Keying } \\ \text { BV } & \text { Bandwidth-Variable } \\ \text { BV-WXC } & \text { Bandwidth-Variable Wavelength Cross-connect } \\ \text { CD } & \text { Chromatic Dispersion } \\ \text { CO-OFDM } & \text { Coherent Optical OFDM } \\ \text { CSRZ } & \text { Carrier-Suppressed Return-to-Zero } \\ \text { DAB } & \text { Digital Audio Broadcasting } \\ \text { DAC } & \text { Digital-to-Analog Convertor } \\ \text { DFT } & \text { Discrete Fourier Transform } \\ \text { DP } & \text { Dual Polarization } \\ \text { DQPSK } & \text { Differential Quadrature Phase-Shift Keying } \\ \text { DSP } & \text { Digital Signal Processing } \\ \text { DT } & \text { Deutsche Telekom } \\ \text { DVB } & \text { Digital Video Broadcasting } \\ \text { EDC } & \text { Electrical Dispersion Compensation } \\ \text { FFT } & \text { Fast Fourier Transform } \\ \text { GB } & \text { Guard Band } \\ \text { GI } & \text { Guard Interval } \\ \text { ICI } & \text { Inter-carrier Interference } \\ \text { IDFT } & \text { Inverse Discrete Fourier Transform } \\ \text { IFFT } & \text { Inverse Fast Fourier Transform } \\ \text { ILP } & \text { Integer Linear Program } \\ \text { IPTV } & \text { Internet Protocol television } \\ \text { IQ } & \text { In-phase and quadrature phase } \\ \text { ISI } & \text { Intersymbol Interference } \\ \text { ITU-T } & \text { International Telecommunication Union-Telecommunication } \\ & \text { Standardization Sector } \\ \text { LA } & \text { Link Aggregation } \\ \text { LCOS } & \text { Liquid Crystal on Silicon } \\ \text { LPF } & \text { Low-Pass Filter } \\ \text { MCM } & \text { Multi-Carrier Modulation } \\ \text { MEMS } & \text { Micro-Electro Mechanical System } \\ & \end{array}$

批注 [gyzhang38]: [Authors Comments] Added according to reviewer1's comments. 


\begin{tabular}{|c|c|}
\hline MILP & Mixed Integer Linear Program \\
\hline MIMO & Multiple-Input Multiple-Output \\
\hline MZM & Mach-Zehnder Modulator \\
\hline No-GI CO-OFDM & No-Guard-Interval CO-OFDM \\
\hline NRZ-OOK & Not Return to Zero On-Off Keying \\
\hline OADM & Optical Add-Drop Multiplexer \\
\hline OFDM & Orthogonal Frequency-Division Multiplexing \\
\hline OWC & Optical Wireless Communication \\
\hline PAPR & Peak-to-Average Power Ratio \\
\hline PDM & Polarization-Division Multiplexing \\
\hline PMD & Polarization Mode Dispersion \\
\hline $\mathrm{P} / \mathrm{S}$ & Parallel/Serial \\
\hline QAM & Quadrature Amplitude Modulation \\
\hline QPSK & Quadrature Phase-Shift Keying \\
\hline RGI-CO-OFDM & Reduced-Guard-Interval CO-OFDM \\
\hline RF & Radio Frequency \\
\hline ROADM & Reconfigurable Optical Add-Drop Multiplexer \\
\hline RSA & Routing and Spectrum Allocation \\
\hline RWA & Routing and Wavelength Assignment \\
\hline SLA & Service Level Agreement \\
\hline SONET & Synchronous Optical Network \\
\hline $\mathrm{S} / \mathrm{P}$ & Serial/Parallel \\
\hline SSMF & Standard Single-Mode Fiber \\
\hline TDM & Time-Division Multiplexing \\
\hline TS & Training Symbols \\
\hline UNI & User-Network Interface \\
\hline WDM & Wavelength-Division Multiplexing \\
\hline WSS & Wavelength Selective Switch \\
\hline WXC & Wavelength Cross-Connect \\
\hline ZGI-CO-OFDM & Zero-Guard-Interval CO-OFDM \\
\hline
\end{tabular}

批注 [gyzhang39]: [Authors

Comments] Added according to

reviewer2's comments.

Comments] Added according to reviewer2's comments. 
Reviewer2/Comment5.3) As a major candidate of the next generation optical network structure, it will be interesting to see the co mpatibility of PON network working with O-OFDM, etc. [3] Cost-effective 33-Gbps intensity modulation direct detection multiband OFDM LR-PON system employing a 10-GHz-based transceiver .

[Authors' Response:] We are aware of the trend of adopting O-OFDM technology in PON. We have added a reference to the suggested paper in the introduction part of Section 3. Because the scope of this survey is focusing on core optical network (as mentioned in the last paragraph in Section 1, and as reflected in the updated title), we did not describe OFDM-PON technology in detail.

\section{页 7: [2] 批注 [gyzhang10]}

Guoying Zhang

2011-12-4 10:20:00

Reviewer2/Comment5.4) Although this paper is focusing on introducing the optical networking with single-mode fiber as data transmission media, the increasing interests in optical wireless communication (OWC) cannot be neglected. The O-OFDM on OWC has different features and requirements than normal fibers, such as the clipping distortion problem. It will be more comprehensive for this paper to briefly cover some related content here. [Authors' Response] We have mentioned OWC as one of the applications of O-OFDM in the beginning of Section 3. Similar to the reason mentioned in the above comment, we didn't cover OWC in detail because of limitationsin scope. 